\title{
Critical mass for a Patlak-Keller-Segel model with degenerate diffusion in higher dimensions
}

\author{
Adrien Blanchet; José A. Carrillo ${ }^{\dagger} \&$ Philippe Laurençot ${ }^{\ddagger}$
}

June 19, 2008

\begin{abstract}
This paper is devoted to the analysis of non-negative solutions for a generalisation of the classical parabolic-elliptic Patlak-Keller-Segel system with $d \geq 3$ and porous medium-like non-linear diffusion. Here, the non-linear diffusion is chosen in such a way that its scaling and the one of the Poisson term coincide. We exhibit that the qualitative behaviour of solutions is decided by the initial mass of the system. Actually, there is a sharp critical mass $M_{c}$ such that if $M \in\left(0, M_{c}\right]$ solutions exist globally in time, whereas there are blowing-up solutions otherwise. We also show the existence of self-similar solutions for $M \in\left(0, M_{c}\right)$. While characterising the possible infinite time blowing-up profile for $M=M_{c}$, we observe that the long time asymptotics are much more complicated than in the classical Patlak-Keller-Segel system in dimension two.
\end{abstract}

\section{Introduction}

In this work, we analyse qualitative properties of non-negative solutions for the Patlak-KellerSegel system in dimension $d \geq 3$ with homogeneous non-linear diffusion given by

$$
\left\{\begin{aligned}
\frac{\partial u}{\partial t}(t, x) & =\operatorname{div}\left[\nabla u^{m}(t, x)-u(t, x) \nabla \phi(t, x)\right] & & t>0, x \in \mathbb{R}^{d}, \\
-\Delta \phi(t, x) & =u(t, x), & & t>0, x \in \mathbb{R}^{d}, \\
u(0, x) & =u_{0}(x) & & x \in \mathbb{R}^{d} .
\end{aligned}\right.
$$

This system has been proposed as a model for chemotaxis-driven cell movement and in the study of a large ensemble of self-gravitating interacting particles, see $[20,15]$ and the literature therein. The purpose of non-linear diffusion with $m>1$ in chemotaxis is to model the local repulsion of cells, see for instance $[37,38]$. This can be interpreted as taking into account anti-crowding effects $[8,38]$.

Initial data will be assumed throughout this paper to verify

$$
u_{0} \in \mathrm{L}^{1}\left(\mathbb{R}^{d} ;\left(1+|x|^{2}\right) \mathrm{d} x\right) \cap \mathrm{L}^{\infty}\left(\mathbb{R}^{d}\right), \quad \nabla u_{0}^{m} \in \mathrm{L}^{2}\left(\mathbb{R}^{d}\right) \quad \text { and } \quad u_{0} \geq 0 .
$$

*DAMTP, Centre for Mathematical Sciences, Wilberforce Road, Cambridge CB3 0WA, United Kingdom. Email: blanchet@ceremade.dauphine.fr, Internet: http://www.ceremade.dauphine.fr/ blanchet/

${ }^{\dagger}$ ICREA (Institució Catalana de Recerca i Estudis Avançats) and Departament de Matemàtiques, Universitat Autònoma de Barcelona, E-08193 Bellaterra, Spain. E-mail: carrillo@mat.uab.es, Internet: http://kinetic.mat.uab.es/ carrillo/

${ }^{\ddagger}$ Institut de Mathématiques de Toulouse, CNRS UMR 5219 \& Université de Toulouse, 118 route de Narbonne, F-31062 Toulouse Cédex 9, France. E-mail: laurenco@mip.ups-tlse.fr, Internet: http://www.mip.upstlse.fr/ laurenco/ 
A fundamental property of the solutions to (1.1) is the formal conservation of the total mass of the system

$$
M:=\int_{\mathbb{R}^{d}} u_{0}(x) \mathrm{d} x=\int_{\mathbb{R}^{d}} u(t, x) \mathrm{d} x \text { for } t \geq 0 .
$$

As the solution to the Poisson equation $-\Delta \phi=u$ is given up to an harmonic function, we choose the one given by $\phi=\mathcal{K} * u$ with

$$
\mathcal{K}(x)=c_{d} \frac{1}{|x|^{d-2}} \quad \text { and } \quad c_{d}:=\frac{1}{(d-2) \sigma_{d}}
$$

where $\sigma_{d}:=2 \pi^{d / 2} / \Gamma(d / 2)$ is the surface area of the sphere $\mathbb{S}^{d-1}$ in $\mathbb{R}^{d}$. The system can thus be rewritten as:

$$
\left\{\begin{aligned}
\frac{\partial u}{\partial t}(t, x) & =\operatorname{div}\left[\nabla u^{m}(t, x)-u(t, x) \nabla(\mathcal{K} * u)(t, x)\right] & & t>0, x \in \mathbb{R}^{d} \\
u(0, x) & =u_{0}(x) \geq 0 & & x \in \mathbb{R}^{d} .
\end{aligned}\right.
$$

In any dimension $d \geq 3$ we will concentrate on a particular choice of the non-linear diffusion exponent

$$
m=m_{d}=: \frac{2(d-1)}{d} \in(1,2) .
$$

This exponent produces an exact balance in the mass-invariant scaling of diffusion and potential drift in (1.3): Indeed, let $u_{\lambda}(x):=\lambda^{d} u(t, \lambda x)$ with $\lambda>0$ of same mass as $u$, the diffusion term $\lambda^{d m+2} \Delta\left(u_{\lambda}^{m}\right)(t, \lambda x)$ has the same scaling as the interaction term $\lambda^{2 d} \operatorname{div}\left(u_{\lambda} \nabla\left(\mathcal{K} * u_{\lambda}\right)\right)(t, \lambda x)$ if and only if $d m+2=2 d$ or equivalently $m=m_{d}$. This balance will appear clearly in the selfsimilar change of variables, see (5.1) below. It can also be understood by the natural Liapunov functional of (1.3), see Lemma 3.1. As we are only interested here in this critical exponent case we will omit, for notational convenience, the index in $m_{d}$ and simply write $m$.

Note that the case $d=2$ and $m_{2}=1$ corresponds to the Patlak-Keller-Segel (PKS) system or to the classical Smoluchowski-Poisson system in two dimensions with linear diffusion [32, 22]. In this case, a simple dichotomy result has been shown in $[17,7]$, namely, the behaviour of the solutions is just determined by the initial mass of the system. More precisely, there exists a critical value of the mass $M_{c}:=8 \pi$ such that if $0<M<M_{c}$ (sub-critical case) the solutions exist globally and if $M>M_{c}$ (super-critical case) the solutions blow up in finite time. Moreover, in the sub-critical case solutions behave self-similarly as $t \rightarrow \infty[4,7]$. Finally, the critical case $M=M_{c}$ was studied in [6] showing that solutions exist globally and blow up as a Dirac mass at the centre of mass as $t \rightarrow \infty$. Solutions have to be understood as free energy solutions, concept that we will specify below.

In this work, we will show that a similar situation to the classical PKS system in $d=2$, although with some important differences, happens for the critical variant of the PKS model in any dimension $d \geq 3$. The main tool for the analysis of this equation is the following free energy functional:

$$
\begin{aligned}
t \mapsto \mathcal{F}[u(t)]: & =\int_{\mathbb{R}^{d}} \frac{u^{m}(t, x)}{m-1}-\frac{1}{2} \iint_{\mathbb{R}^{d} \times \mathbb{R}^{d}} \mathcal{K}(x-y) u(t, x) u(t, y) \mathrm{d} x \mathrm{~d} y \\
& =\int_{\mathbb{R}^{d}} \frac{u^{m}(t, x)}{m-1}-\frac{c_{d}}{2} \iint_{\mathbb{R}^{d} \times \mathbb{R}^{d}} \frac{1}{|x-y|^{d-2}} u(t, x) u(t, y) \mathrm{d} x \mathrm{~d} y
\end{aligned}
$$

which is related to its time derivative, the Fisher information, in the following way: given a smooth positive fast-decaying solution to (1.3), then

$$
\frac{\mathrm{d}}{\mathrm{d} t} \mathcal{F}[u(t)]=-\int_{\mathbb{R}^{d}} u(t, x)\left|\nabla\left(\frac{m}{m-1} u^{m-1}(t, x)-\phi(t, x)\right)\right|^{2} \mathrm{~d} x .
$$


We will give a precise sense to this entropy/entropy-dissipation relation below.

The system (1.3) can formally be considered a particular instance of the general family of partial differential equations studied in $[11,1,12]$. The free energy functional $\mathcal{F}$ structurally belongs to the general class of free energies for interacting particles introduced in $[28,11,12]$. The functionals treated in those references are of the general form:

$$
\mathcal{E}[n]:=\int_{\mathbb{R}^{d}} U[n(x)] \mathrm{d} x+\int_{\mathbb{R}^{d}} n(x) V(x) \mathrm{d} x+\frac{1}{2} \iint_{\mathbb{R}^{d} \times \mathbb{R}^{d}} W(x-y) n(x) n(y) \mathrm{d} x \mathrm{~d} y
$$

under the basic assumptions $U: \mathbb{R}^{+} \rightarrow \mathbb{R}$ is a density of internal energy, $V: \mathbb{R}^{d} \rightarrow \mathbb{R}$ is a convex smooth confinement potential and $W: \mathbb{R}^{d} \rightarrow \mathbb{R}$ is a symmetric convex smooth interaction potential. The internal energy $U$ should satisfy the following dilation condition, introduced in McCann [28]

$$
\lambda \longmapsto \lambda^{d} U\left(\lambda^{-d}\right) \quad \text { is convex non-increasing on } \mathbb{R}^{+} .
$$

In fact, the free energy functional plays a central role for this equation since (1.3) can be formally considered as a gradient flow of the free energy functional with respect to the euclidean transport distance like for the porous medium equation [31]. In our case, the interaction potential is singular and the key tool of displacement convexity of the functional fails, making the theory in the previous references not useful for our purposes, as in [5]. Before proceeding further, let us state the notion of solutions, in this paper we will deal with :

Definition 1.1 (Weak and free energy solution) Let $u_{0}$ be an initial condition satisfying (1.2) and $T \in(0, \infty]$.

(i) A weak solution to (1.3) on $[0, T)$ with initial condition $u_{0}$ is a non-negative function $u \in C\left([0, T) ; \mathrm{L}^{1}\left(\mathbb{R}^{d}\right)\right)$ such that $\left.u \in \mathrm{L}^{\infty}\left((0, t) \times \mathbb{R}^{d}\right)\right), u^{m} \in \mathrm{L}^{2}\left(0, t ; H^{1}\left(\mathbb{R}^{d}\right)\right)$ for each $t \in[0, T)$ and

$$
\begin{array}{r}
\int_{\mathbb{R}^{d}} u_{0}(x) \psi(0, x) \mathrm{d} x=\int_{0}^{T} \int_{\mathbb{R}^{d}}\left[\nabla u^{m}(t, x)-u(t, x) \nabla \phi(t, x)\right] \cdot \nabla \psi(t, x) \mathrm{d} x \mathrm{~d} t \\
-\int_{0}^{T} \int_{\mathbb{R}^{d}} u(t, x) \partial_{t} \psi(t, x) \mathrm{d} x \mathrm{~d} t
\end{array}
$$

for any test function $\psi \in \mathcal{D}\left([0, T) \times \mathbb{R}^{d}\right)$ with $\phi=\mathcal{K} * u$.

(ii) $A$ free energy solution to (1.3) on $[0, T)$ with initial condition $u_{0}$ is a weak solution $u$ to (1.3) on $[0, T)$ with initial condition $u_{0}$ satisfying additionally: $u^{(2 m-1) / 2} \in \mathrm{L}^{2}\left(0, t ; H^{1}\left(\mathbb{R}^{d}\right)\right)$ and

$$
\mathcal{F}[u(t)]+\int_{0}^{t} \int_{\mathbb{R}^{d}}\left|\left(\frac{2 m}{2 m-1} \nabla u^{(2 m-1) / 2}(s, x)-u^{1 / 2}(s, x) \nabla \phi(s, x)\right)\right|^{2} \mathrm{~d} x \mathrm{~d} s \leq \mathcal{F}\left[u_{0}\right]
$$

for all $t \in(0, T)$ with $\phi=\mathcal{K} * u$.

In (1.6), we cannot write the Fisher information factorised by $u$ as in (1.4) because of the lack of regularity of $u$. We note that both (1.5) and (1.6) are meaningful. Indeed, the regularity required for $u$ implies that the solution $\phi=\mathcal{K} * u$ to the Poisson equation satisfies $\phi \in \mathrm{L}^{\infty}\left(0, t ; H^{1}\left(\mathbb{R}^{d}\right)\right)$ for all $t \in(0, T)$. In addition, it follows from (1.5) by classical approximation arguments that

$$
\|u(t)\|_{1}=\int_{\mathbb{R}^{d}} u(t, x) \mathrm{d} x=\int_{\mathbb{R}^{d}} u_{0}(x) \mathrm{d} x=\left\|u_{0}\right\|_{1}=M \quad \text { for } \quad t \in[0, T) .
$$


Indeed, take a sequence of functions $\left(\psi_{R}\right)$ in $\mathcal{D}\left([0, T) \times \mathbb{R}^{d}\right)$ such that $\psi_{R} \longrightarrow \mathbf{1}_{[0, t]}$ a.e. and $0 \leq \psi_{R} \leq 1$. Writing (1.5) with $\psi=\psi_{R}$, the integrability properties of $u, \nabla u^{m}$ and $\nabla \phi$ allow us to pass to the limit as $R \rightarrow \infty$ and deduce (1.7).

Let us point out that the existence of free energy solutions for a related problem was essentially obtained in $[34,35,30]$ where the Poisson equation is replaced by $-\Delta \phi=u-\phi$. There, the authors also show that the mass is the suitable quantity for (1.3) allowing for a dichotomy. Precisely, the author shows that there exist two masses $0<M_{1}<M_{2}$ such that if $0<M<M_{1}$ the solutions exist globally in time, while for $M>M_{2}$ there are solutions blowing up in finite time. The values of these masses, are related to the sharp constants of the Sobolev inequality.

Here, we will make a fundamental use of a variant to the Hardy-Littlewood-Sobolev (VHLS) inequality, see Lemma 3.2: for all $h \in \mathrm{L}^{1}\left(\mathbb{R}^{d}\right) \cap \mathrm{L}^{m}\left(\mathbb{R}^{d}\right)$, there exists an optimal constant $C_{*}$ such that

$$
\left|\iint_{\mathbb{R}^{d} \times \mathbb{R}^{d}} \frac{h(x) h(y)}{|x-y|^{d-2}} \mathrm{~d} x \mathrm{~d} y\right| \leq C_{*}\|h\|_{m}^{m}\|h\|_{1}^{2 / d} .
$$

This inequality will play the same role as the logarithmic HLS inequality proved in [9] for the classical PKS system in $d=2[17,7,6]$. The VHLS inequality and the identification of the equality cases allow us to give the first main result of this work, namely, the following sharp critical mass

$$
M_{c}:=\left[\frac{2}{(m-1) C_{*} c_{d}}\right]^{d / 2}
$$

for equation (1.3). More precisely, we will show that free energy solutions exist globally for $M \in\left(0, M_{c}\right]$ while there are finite time blowing-up solutions otherwise. However, the long time asymptotics of the solutions is much more complicated compared to the classical PKS system in two dimensions. The main results of this work and the open problems related to large times asymptotics can be summarised as follows:

- Sub-critical case: $0<M<M_{c}$, solutions exist globally in time and there exists a radially symmetric compactly supported self-similar solution, although we are not able to show that it attracts all global solutions. See Proposition 4.4, Theorem 5.2 and Corollary 5.7.

- Critical case: $M=M_{c}$, solutions exist globally in time, see Proposition 4.7. There are infinitely many compactly supported stationary solutions. The second moment of solutions is non-decreasing in time, with two possibilities we cannot exclude: either is uniformly bounded in time or diverges. Moreover, the $\mathrm{L}^{m}$-norm of the solution could be divergent as $t \rightarrow \infty$ or a diverging sequence of times could exist with bounded $\mathrm{L}^{m}$-norm. However, we show a striking difference with respect to the classical PKS system in two dimensions [6], namely, the existence of global in time solutions not blowing-up in infinite time. We will comment further on these issues in Section 4.2.3.

- Super-critical case: $M>M_{c}$, we prove that there exist solutions, corresponding to initial data with negative free energy, blowing up in finite time, see Proposition 4.3. However, we cannot exclude the possibility that solutions with positive free energy may be global in time.

This model shares with the non-linear Schrödinger equation (NLS) $[14,36]$ and the unstable thin-film equation (UTF) [2,3] a very rich behaviour with two "levels of criticality". The first level is given by the homogeneity of the "attractive" and "repulsive" terms in each problem. In our particular case, this refers to the aggregation versus diffusion mechanisms. As seen above, the balance happens precisely for our chosen exponent $m=m_{d}$. In the NLS equation this happens for the so-called pseudo-conformal nonlinearity [14, Chapter 6]. In the UTF equation this happens in the so-called marginal case $[2,3]$. Our paper is concerned with the second level of criticality 
which only occurs when the homogeneity of the "attractive" and "repulsive" terms matches. In that particular case and for the three models there exists a critical value $M_{c}$ of the mass which is the maximum value of the mass below which the solutions exist globally in time, see [14, 39, 29] for the pseudo-conformal NLS equation and $[3,33]$ for the marginal UTF equation. Note that mass refers to the $L^{2}$-norm of the solution for the NLS equation and the $L^{1}$-norm for (1.3) and the UTF equation.

Let us also point out that in all these three problems, the virial method is an elegant way to prove that there are solutions which blow-up above the critical mass, see Remark 4.2. A common technique with the NLS equation is the use of the concentration compactness method for clarifying the blow-up scenario for critical mass, see Proposition 4.5. For the NLS equation, the result in [29] goes further and clarifies the blow-up for super-critical masses close to critical. Moreover, all these models have in common the existence of suitable free energies with two competing terms. As a main difference the free energy of the NLS equation is conservative whereas it is dissipated for (1.3) and the UTF equation.

The results are organised as follows. Section 2 shows a key maximal time of existence criterion for free energy solutions of equation (1.3). This criterion improves over the results in $[34,35]$ since it is only based on the boundedness or unboundedness in time of the $\mathrm{L}^{m}$-norm of the solutions and it has to be compared to a similar criterion based on the logarithmic entropy in the classical PKS system in two dimensions obtained in [6]. Section 3 is devoted to the variational study of the minimisation of the free energy functional over the set of densities with a fixed mass. With that aim the proof of the VHLS inequality and the identification of the equality cases are performed. Section 4 uses this variational information to show the above main results concerning the dichotomy, the global existence for $M<M_{c}$ and the characterisation by concentrationcompactness techniques of the nature of the possible blow-up in the critical case leading to the global existence for this critical value. Finally, the last section is devoted to the study of the free energy functional in self-similar variables and the proof of the existence of self-similar solutions in the sub-critical case.

\section{Existence criterion}

As in $[34,35]$, we consider the regularised problem

$$
\left\{\begin{aligned}
\frac{\partial u_{\varepsilon}}{\partial t}(t, x) & =\operatorname{div}\left[\nabla\left(f_{\varepsilon} \circ u_{\varepsilon}\right)(t, x)-u_{\varepsilon}(t, x) \nabla \phi_{\varepsilon}(t, x)\right] & & t>0, x \in \mathbb{R}^{d}, \\
\phi_{\varepsilon}(t, x) & =\mathcal{K} * u_{\varepsilon}(t, x), & & t>0, x \in \mathbb{R}^{d}, \\
u_{\varepsilon}(0, x) & =u_{0}^{\varepsilon} \geq 0 & & x \in \mathbb{R}^{d},
\end{aligned}\right.
$$

where $f_{\varepsilon}:[0, \infty) \longrightarrow \mathbb{R}$ is given by $f_{\varepsilon}(u):=(u+\varepsilon)^{m}-\varepsilon^{m}$. Here, $u_{0}^{\varepsilon}$ is the convolution of $u_{0}$ with a sequence of mollifiers and $\left\|u_{0}^{\varepsilon}\right\|_{1}=\left\|u_{0}\right\|_{1}=M$ in particular. This regularised problem has global in time smooth solutions. This approximation has been proved to be convergent. More precisely, the result in [35, Section 4] asserts that if we assume that

$$
\sup _{0<t<T}\left\|u_{\varepsilon}(t)\right\|_{\infty} \leq \kappa
$$

where $\kappa$ is independent of $\varepsilon>0$, then there exists a sub-sequence $\varepsilon_{n} \rightarrow 0$, such that

$$
\begin{array}{rll}
u_{\varepsilon_{n}} \rightarrow u & \text { strongly } & \text { in } C\left([0, T], \mathrm{L}_{\text {loc }}^{p}\left(\mathbb{R}^{d}\right)\right) \text { and a.e. in }(0, T) \times \mathbb{R}^{d}, \\
\nabla u_{\varepsilon_{n}}^{m} \rightarrow \nabla u^{m} & \text { weakly-* } & \text { in } \mathrm{L}^{\infty}\left(0, T ; \mathrm{L}^{2}\left(\mathbb{R}^{d}\right)\right), \\
\phi_{\varepsilon_{n}}(t) \rightarrow \phi(t) & \text { strongly } & \text { in } \mathrm{L}_{\text {loc }}^{r}\left(\mathbb{R}^{d}\right) \text { a.e. in }(0, T), \\
\nabla \phi_{\varepsilon_{n}}(t) \rightarrow \nabla \phi(t) & \text { strongly } & \text { in } \mathrm{L}_{\text {loc }}^{r}\left(\mathbb{R}^{d}\right) \text { a.e. in }(0, T),
\end{array}
$$


for any $p \in(1, \infty)$ and $r \in(1, \infty]$, and $u$ is a weak solution to $(1.3)$ on $[0, T)$ with $\phi=\mathcal{K} * u$. Moreover, the free energy [34, Proposition 6.1] satisfies $\mathcal{F}[u(t)] \leq \mathcal{F}\left[u_{0}\right]$ for a.e. $t \geq 0$. However, a detailed analysis of the proof in [34, Proposition 6.1] shows that the weak solution is in fact a free energy solution.

Proposition 2.1 (Existence of free energy solutions) Under assumption (1.2) on the initial data and (2.2) on the approximation sequence, there exists a free energy solution to (1.3) in $[0, T)$.

Proof. The only remaining points not covered by the results in $[34,35]$ are the lower semicontinuity of the free energy dissipation and the fact that $u^{(2 m-1) / 2}$ belongs to $L^{2}\left(0, t ; H^{1}\left(\mathbb{R}^{d}\right)\right)$ for $t \in[0, T)$. The latter will actually be shown in the proof of Lemma 2.3, see (2.9) below. Concerning the former, a careful reading of the proof of [34, Proposition 6.1] gives that

$\mathcal{F}_{\varepsilon, l}\left[u_{\varepsilon}(t)\right]+\frac{3}{4} \int_{0}^{t} \int_{\mathbb{R}^{d}}\left[u_{\varepsilon}(s, x)+\varepsilon\right]\left|\nabla\left(\frac{m}{m-1}\left[u_{\varepsilon}(s, x)+\varepsilon\right]^{m-1}-\phi_{\varepsilon}(s, x)\right)\right|^{2} \psi_{l}(x) \mathrm{d} x \mathrm{~d} t \leq \mathcal{F}_{\varepsilon, l}\left[u_{0}\right]$

for a.e. $t \in(0, T)$ where $\psi_{l}$ is a standard cut-off function in $\mathbb{R}^{d}$ for any $l \in \mathbb{N}$ and

$$
\mathcal{F}_{\varepsilon, l}\left[u_{\varepsilon}(t)\right]=\int_{\mathbb{R}^{d}} \frac{\left[u_{\varepsilon}(t, x)+\varepsilon\right]^{m}}{m-1} \psi_{l}(x) \mathrm{d} x-\frac{1}{2} \iint_{\mathbb{R}^{d} \times \mathbb{R}^{d}} \mathcal{K}(x-y) u_{\varepsilon}(t, x) u_{\varepsilon}(t, y) \mathrm{d} x \mathrm{~d} y .
$$

In this regularised setting, we can write that

$$
\left(u_{\varepsilon}+\varepsilon\right)\left|\nabla\left[\frac{m}{m-1}\left(u_{\varepsilon}+\varepsilon\right)^{m-1}-\phi_{\varepsilon}\right]\right|^{2}=\left|\frac{2 m}{2 m-1} \nabla\left(u_{\varepsilon}+\varepsilon\right)^{(2 m-1) / 2}-\left(u_{\varepsilon}+\varepsilon\right)^{1 / 2} \nabla \phi_{\varepsilon}\right|^{2} .
$$

As proved in [34], we have $\mathcal{F}_{\varepsilon_{n}, l}\left[u_{\varepsilon_{n}}(t)\right] \rightarrow \mathcal{F}[u(t)]$ as $\varepsilon_{n} \rightarrow 0$ and $l \rightarrow \infty$. In addition, it is straightforward from the convergence properties (2.3)-(2.6) above to pass to the limit as $\varepsilon_{n} \rightarrow 0$ in the free energy dissipation functional with the help of a lower semi-continuity argument. We leave the details to the reader, see e.g. [31] or [10, Lemma 10]. Hence, passing to the limit as $l \rightarrow \infty$, then $u$ is a free energy solution as it satisfies the free energy inequality (1.6).

Remark 2.2 The free energy inequality (1.6) can be obtained with constant $3 / 4$ multiplying the entropy dissipation directly from [34, Proposition 6.1] and the procedure above. This is a technical issue that can be improved to constant 1 by redoing the proof in [34, Proposition 6.1] treating more carefully the free energy dissipation term. In fact, the proof in [34, Proposition 6.1] shows that one can choose the constant as close to 1 as wanted.

According to Proposition 2.1, the existence of a free energy solution to $(1.3)$ on $[0, T)$ depends on the availability of the $L^{\infty}$-bound (2.2) and we shall focus on establishing the latter. As we shall see in the next lemma, such a bound actually follows from a similar bound in $L^{m}\left(\mathbb{R}^{d}\right)$ which additionally provides a characterisation of the maximal existence time. More precisely, we characterise the maximal time of existence by showing the local in time boundedness of the $L^{m}$-norm independently of the approximation parameter $\varepsilon>0$ and how this estimate implies the local in time $L^{\infty}$-estimate $(2.2)$.

Lemma 2.3 (From uniform integrability to $\mathrm{L}^{\infty}$-bounds) For any $\eta>0$ there exists $\tau_{\eta}>$ 0 depending only on $d, M$, and $\eta$ such that, if

$$
\sup _{\varepsilon \in(0,1)}\left\|u_{\varepsilon}\left(t^{*}\right)\right\|_{m} \leq \eta
$$

for some $t^{*} \in[0, \infty)$, then 
(i) the family $\left(u_{\varepsilon}\right)_{\varepsilon}$ is bounded in $\mathrm{L}^{\infty}\left(t^{*}, t^{*}+\tau_{\eta} ; \mathrm{L}^{m}\left(\mathbb{R}^{d}\right)\right)$.

(ii) Moreover, if $\left(u_{\varepsilon}\left(t^{*}\right)\right)_{\varepsilon}$ is also bounded in $\mathrm{L}^{p}\left(\mathbb{R}^{d}\right)$ for some $p \in(m, \infty]$, then $\left(u_{\varepsilon}\right)_{\varepsilon}$ is bounded in $\mathrm{L}^{\infty}\left(t^{*}, t^{*}+\tau_{\eta} ; \mathrm{L}^{p}\left(\mathbb{R}^{d}\right)\right)$.

Proof. To prove this result we need to refine the argument already used in the two-dimensional situation $d=2$ with linear diffusion $m=1$ in $[7,6]$. We follow a procedure analogous to the ones in $[23,8,34,16]$.

Step 1 - $L^{m}$-estimates: By (2.1) we have

$$
\begin{aligned}
\frac{\mathrm{d}}{\mathrm{d} t}\left\|u_{\varepsilon}\right\|_{m}^{m} & =-m(m-1) \int_{\mathbb{R}^{d}} u_{\varepsilon}^{m-2} \nabla u_{\varepsilon} \cdot\left(m\left(u_{\varepsilon}+\varepsilon\right)^{m-1} \nabla u_{\varepsilon}-u_{\varepsilon} \nabla \phi_{\varepsilon}\right) \mathrm{d} x \\
& \leq-\frac{4 m^{2}(m-1)}{(2 m-1)^{2}}\left\|\nabla u_{\varepsilon}^{(2 m-1) / 2}\right\|_{2}^{2}-(m-1) \int_{\mathbb{R}^{d}} u_{\varepsilon}^{m} \Delta \phi_{\varepsilon} \mathrm{d} x \\
& =-\frac{4 m^{2}(m-1)}{(2 m-1)^{2}}\left\|\nabla u_{\varepsilon}^{(2 m-1) / 2}\right\|_{2}^{2}+(m-1)\left\|u_{\varepsilon}\right\|_{m+1}^{m+1} .
\end{aligned}
$$

As

$$
1<\frac{2 m}{2 m-1}<\frac{2(m+1)}{2 m-1}<\frac{2 d}{d-2},
$$

we have the following Gagliardo-Nirenberg-Sobolev inequality: there exists a positive constant $C$ such that

$$
\|w\|_{2(m+1) /(2 m-1)} \leq C\|\nabla w\|_{2}^{[(2 m-1) d] /[(m+1)(2 m+d-2)]}\|w\|_{2 m /(2 m-1)}^{2 m^{2} /[(m+1)(2 m+d-2)]}
$$

which we apply with $w=u_{\varepsilon}^{(2 m-1) / 2}$ to obtain

$$
\left\|u_{\varepsilon}\right\|_{m+1}^{(2 m-1) / 2} \leq C\left\|\nabla u_{\varepsilon}^{(2 m-1) / 2}\right\|_{2}^{[(2 m-1) d] /[(m+1)(2 m+d-2)]}\left\|u_{\varepsilon}\right\|_{m}^{m^{2}(2 m-1) /[(m+1)(2 m+d-2)]} .
$$

It leads to

$$
\begin{aligned}
\left\|u_{\varepsilon}\right\|_{m+1}^{m+1} & \leq C\left\|\nabla u_{\varepsilon}^{(2 m-1) / 2}\right\|_{2}^{2 d /(2 m+d-2)}\left\|u_{\varepsilon}\right\|_{m}^{2 m^{2} /(2 m+d-2)} \\
& \leq \frac{2 m^{2}}{(2 m-1)^{2}}\left\|\nabla u_{\varepsilon}^{(2 m-1) / 2}\right\|_{2}^{2}+C\left\|u_{\varepsilon}\right\|_{m}^{m^{2} /(m-1)} .
\end{aligned}
$$

We thus end up with

$$
\frac{\mathrm{d}}{\mathrm{d} t}\left\|u_{\varepsilon}\right\|_{m}^{m}+\frac{2 m^{2}(m-1)}{(2 m-1)^{2}}\left\|\nabla u_{\varepsilon}^{(2 m-1) / 2}\right\|_{2}^{2} \leq(m-1) C\left\|u_{\varepsilon}\right\|_{m}^{m^{2} /(m-1)} .
$$

In particular, for any $t_{2} \geq t_{1} \geq 0$

$$
\left\|u_{\varepsilon}\left(t_{2}\right)\right\|_{m}^{m} \leq\left[\left\|u_{\varepsilon}\left(t_{1}\right)\right\|_{m}^{-m /(m-1)}-C\left(t_{2}-t_{1}\right)\right]^{-(m-1)}
$$

Taking $t_{1}=t^{*}$ we deduce from (2.8) that

$$
\left\|u_{\varepsilon}(t)\right\|_{m}^{m} \leq\left(\eta^{-m /(m-1)}-C\left(t-t^{*}\right)\right)^{-(m-1)} \quad \text { for } t \in\left[t^{*}, t^{*}+2 \tau_{\eta}\right)
$$

with $\tau_{\eta}=1 /\left(2 C \eta^{m /(m-1)}\right)$. Consequently, $\left\|u_{\varepsilon}(t)\right\|_{m}^{m} \leq\left(C \tau_{\eta}\right)^{-(m-1)}$ for $t \in\left[t^{*}, t^{*}+\tau_{\eta}\right]$ and the proof of the first assertion of Lemma 2.3 is complete. In addition, coming back to (2.7), we further deduce that

$$
\int_{t^{*}}^{t^{*}+\tau_{\eta}}\left\|\nabla u_{\varepsilon}^{(2 m-1) / 2}\right\|_{2}^{2} \leq C\left(t^{*}, \eta\right)
$$


Step 2 - $L^{p}$-estimates, $p \in(m, \infty):$ For $t \in\left[t^{*}, t^{*}+\tau_{\eta}\right], K \geq 1$, and $p>m$, we infer from (2.1) that

$$
\begin{aligned}
\frac{\mathrm{d}}{\mathrm{d} t}\left\|\left(u_{\varepsilon}(t)-K\right)_{+}\right\|_{p}^{p} \leq & -m p(p-1) \int_{\mathbb{R}^{d}}\left(u_{\varepsilon}(t)-K\right)_{+}^{p-2}\left(u_{\varepsilon}+\varepsilon\right)^{m-1}\left|\nabla u_{\varepsilon}\right|^{2} \mathrm{~d} x \\
& +p(p-1) \int_{\mathbb{R}^{d}}\left[\left(u_{\varepsilon}(t)-K\right)_{+}^{p-1}+K\left(u_{\varepsilon}(t)-K\right)_{+}^{p-2}\right] \nabla u_{\varepsilon} \cdot \nabla \phi_{\varepsilon} \mathrm{d} x \\
\leq & -m p(p-1) \int_{\mathbb{R}^{d}}\left(u_{\varepsilon}(t)-K\right)_{+}^{m+p-3}\left|\nabla u_{\varepsilon}\right|^{2} \mathrm{~d} x \\
& -\int_{\mathbb{R}^{d}}\left[(p-1)\left(u_{\varepsilon}(t)-K\right)_{+}^{p}+p K\left(u_{\varepsilon}(t)-K\right)_{+}^{p-1}\right] \Delta \phi_{\varepsilon} \mathrm{d} x \\
\leq & -\frac{4 m p(p-1)}{(m+p-1)^{2}}\left\|\nabla\left[\left(u_{\varepsilon}(t)-K\right)_{+}^{(m+p-1) / 2}\right]\right\|_{2}^{2}+(\mathrm{I})
\end{aligned}
$$

with

$(\mathrm{I}):=p K^{2}\left\|\left(u_{\varepsilon}(t)-K\right)_{+}\right\|_{p-1}^{p-1}+(2 p-1) K\left\|\left(u_{\varepsilon}(t)-K\right)_{+}\right\|_{p}^{p}+(p-1)\left\|\left(u_{\varepsilon}(t)-K\right)_{+}\right\|_{p+1}^{p+1}$.

We now use the following interpolation inequality

$$
\|w\|_{p+1}^{p+1} \leq C(p)\left\|\nabla\left(w^{(m+p-1) / 2}\right)\right\|_{2}^{2}\|w\|_{1}^{2 / d}
$$

which is a consequence of the Gagliardo-Nirenberg-Sobolev and Hölder inequalities (see, e.g., [35, Lemma 3.2]) to obtain

$$
\begin{aligned}
(\mathrm{I}) & \leq(p-1) K^{2}\left\|\left(u_{\varepsilon}(t)-K\right)_{+}\right\|_{p}^{p}+K^{2}\left|\left\{x: u_{\varepsilon}(t, x) \geq K\right\}\right|+(2 p-1) K\left\|\left(u_{\varepsilon}(t)-K\right)_{+}\right\|_{p}^{p} \\
& +C(p)\left\|\nabla\left[\left(u_{\varepsilon}(t)-K\right)_{+}^{(m+p-1) / 2}\right]\right\|_{2}^{2}\left\|\left(u_{\varepsilon}(t)-K\right)_{+}\right\|_{1}^{2 / d} .
\end{aligned}
$$

Noting that

$$
\left\|\left(u_{\varepsilon}(t)-K\right)_{+}\right\|_{1} \leq\left\|u_{\varepsilon}(t)\right\|_{m}\left(\frac{\left\|u_{\varepsilon}(t)\right\|_{1}}{K}\right)^{(m-1) / m}
$$

and recalling that $\left\|u_{\varepsilon}(t)\right\|_{1}=M$ we conclude that

$$
\begin{aligned}
(\mathrm{I}) & \leq C(p) \frac{\left\|u_{\varepsilon}(t)\right\|_{m}^{2 / d}}{K^{2(m-1) / m d}}\left\|\nabla\left[\left(u_{\varepsilon}(t)-K\right)_{+}^{(m+p-1) / 2}\right]\right\|_{2}^{2} \\
& +K[2 p-1+(p-1) K]\left\|\left(u_{\varepsilon}(t)-K\right)_{+}\right\|_{p}^{p}+K M .
\end{aligned}
$$

By Step 1, we may choose $K=K_{*}$ large enough such that

$$
C \frac{\left\|u_{\varepsilon}(t)\right\|_{m}^{2 / d}}{K_{*}^{2(m-1) / m d}} \leq \frac{4 m p(p-1)}{(m+p-1)^{2}}
$$

for all $t \in\left[t^{*}, t^{*}+\tau_{\eta}\right]$ and $\varepsilon \in(0,1)$, hence

$$
(\mathrm{I}) \leq \frac{4 m p(p-1)}{(m+p-1)^{2}}\left\|\nabla\left[\left(u_{\varepsilon}(t)-K_{*}\right)_{+}^{(m+p-1) / 2}\right]\right\|_{2}^{2}+C\left(p, t^{*}, \eta\right)\left[1+\left\|\left(u_{\varepsilon}(t)-K_{*}\right)_{+}\right\|_{p}^{p}\right] .
$$

Therefore

$$
\frac{\mathrm{d}}{\mathrm{d} t}\left\|\left(u_{\varepsilon}(t)-K_{*}\right)_{+}\right\|_{p}^{p} \leq C\left(p, t^{*}, \eta\right)\left[1+\left\|\left(u_{\varepsilon}(t)-K_{*}\right)_{+}\right\|_{p}^{p}\right]
$$

so that

$$
\left\|\left(u_{\varepsilon}(t)-K_{*}\right)_{+}\right\|_{p}^{p} \leq C\left(p, t^{*}, \eta\right) \quad \text { for } \quad t \in\left[t^{*}, t^{*}+\tau_{\eta}\right] \quad \text { and } \quad \varepsilon \in(0,1)
$$


As

$$
\left\|u_{\varepsilon}(t)\right\|_{p}^{p} \leq C(p)\left(K_{*}^{p-m}\left\|u_{\varepsilon}(t)\right\|_{m}^{m}+\left\|\left(u_{\varepsilon}(t)-K_{*}\right)_{+}\right\|_{p}^{p}\right),
$$

the previous inequality and Step 1 warrant that

$$
\left\|u_{\varepsilon}(t)\right\|_{p} \leq C\left(p, t^{*}, \eta\right) \text { for } t \in\left[t^{*}, t^{*}+\tau_{\eta}\right] \text { and } \varepsilon \in(0,1) .
$$

Step 3 - $\mathrm{L}^{\infty}$-estimates: As a direct consequence of Step 2 with $p=d+1$ and Morrey's embedding theorem $\left(\nabla \phi_{\varepsilon}\right)_{\varepsilon}$ is bounded in $\mathrm{L}^{\infty}\left(\left(t^{*}, t^{*}+\tau_{\eta}\right) \times \mathbb{R}^{d} ; \mathbb{R}^{d}\right)$. This property in turn implies that $\left(u_{\varepsilon}\right)_{\varepsilon}$ is bounded in $\mathrm{L}^{\infty}\left(\left(t^{*}, t^{*}+\tau_{\eta}\right) \times \mathbb{R}^{d}\right)$ and we refer to [8, Lemma 3.2] and [23] for a proof (see also [35, Section 5] and [34, Theorem 1.2] for alternative arguments).

As a consequence of the previous lemma, we are able to construct a free energy solution defined on a maximal existence time.

Theorem 2.4 (Maximal free energy solution) Under assumption (1.2) on the initial condition there are $T_{\omega} \in(0, \infty]$ and a free energy solution $u$ to $(1.3)$ on $\left[0, T_{\omega}\right)$ with the following alternative: Either $T_{\omega}=\infty$ or $T_{\omega}<\infty$ and $\|u(t)\|_{m} \rightarrow \infty$ as $t \nearrow T_{\omega}$. Furthermore there exists a positive constant $C_{0}$ depending only on $d$ such that $u$ satisfies

$$
\left\|u\left(t_{2}\right)\right\|_{m}^{m} \leq\left(\left\|u\left(t_{1}\right)\right\|_{m}^{-m /(m-1)}-C_{0}\left(t_{2}-t_{1}\right)\right)^{-(m-1)}
$$

for $t_{1} \in\left[0, T_{\omega}\right)$ and $t_{2} \in\left(t_{1}, T_{\omega}\right)$.

Proof. We put $\xi_{p}(t)=\sup _{\varepsilon \in(0,1)}\left\|u_{\varepsilon}(t)\right\|_{p} \in(0, \infty]$ for $t \geq 0$ and $p \in[m, \infty]$ and

$$
T_{1}=\sup \left\{T>0: \xi_{m} \in \mathrm{L}^{\infty}(0, T)\right\} .
$$

Clearly the definition of the sequence $\left(u_{0}^{\varepsilon}\right)_{\varepsilon}$ and $(1.2)$ ensure that $\xi_{p}(0)$ is finite for all $p \in[m, \infty]$. By Lemma 2.3 there exists $t_{1}>0$ such that $\xi_{p}$ is bounded on $\left[0, t_{1}\right]$ for all $p \in[m, \infty]$. Then $(2.2)$ is fulfilled for $T=t_{1}$ and there is a free energy solution to $(1.3)$ on $\left[0, t_{1}\right)$ by Proposition 2.1 and (2.9). This ensures in particular that $T_{1} \geq t_{1}>0$.

We next claim that

$$
\xi_{\infty} \in \mathrm{L}^{\infty}(0, T) \text { for any } T \in\left[0, T_{1}\right) .
$$

Indeed, consider $T_{1}^{\infty}=\sup \left\{T \in\left(0, T_{1}\right): \xi_{\infty} \in \mathrm{L}^{\infty}(0, T)\right\}$ and assume for contradiction that $T_{1}^{\infty}<T_{1}$. Then $\xi_{m}$ belongs to $\mathrm{L}^{\infty}\left(0, T_{1}^{\infty}\right)$ and we put $\eta=\left\|\xi_{m}\right\|_{L^{\infty}\left(0, T_{1}^{\infty}\right)}$ and $t^{*}=T_{1}^{\infty}-\left(\tau_{\eta} / 2\right)$, $\tau_{\eta}$ being defined in Lemma 2.3. As $\xi_{m}\left(t^{*}\right) \leq \eta$ and $\xi_{\infty}\left(t^{*}\right)$ is finite we may apply Lemma 2.3 to deduce that both $\xi_{m}$ and $\xi_{\infty}$ belong to $\mathrm{L}^{\infty}\left(t^{*}, t^{*}+\tau_{\eta}\right)$, the latter property contradicting the definition of $T_{1}^{\infty}$ as $t^{*}+\tau_{\eta}=T_{1}^{\infty}+\left(\tau_{\eta} / 2\right)$.

Now, thanks to $(2.11),(2.2)$ is fulfilled for any $T \in\left[0, T_{1}\right)$ and the existence of a free energy solution $u$ to (1.3) on $\left[0, T_{1}\right)$ follows from Proposition 2.1 and (2.9). Moreover, either $T_{1}=\infty$ or $T_{1}<\infty$ and $\|u(t)\|_{m} \rightarrow \infty$ as $t \nearrow T_{1}$, and the proof of Theorem 2.4 is complete with $T_{\omega}=T_{1}$. Or $T_{1}<\infty$ and

$$
\liminf _{t \rightarrow T_{1}}\|u(t)\|_{m}<\infty .
$$

In that case, there are $\eta>0$ and an increasing sequence of positive real numbers $\left(s_{j}\right)_{j \geq 1}$ such that $s_{j} \rightarrow T_{1}$ as $j \rightarrow \infty$ and $\left\|u\left(s_{j}\right)\right\|_{m} \leq \eta$. Fix $j_{0} \geq 1$ such that $s_{j_{0}} \geq T_{1}-\left(\tau_{\eta} / 2\right)$ with $\tau_{\eta}$ defined in Lemma 2.3 and put $\tilde{u}_{0}=u\left(s_{j_{0}}\right)$; According to Definition 1.1 and (2.4) $\tilde{u}_{0}$ fulfils (1.2) and we may proceed as above to obtain a free energy solution $\tilde{u}$ to $(1.3)$ on $\left[0, T_{2}\right)$ for some $T_{2} \geq \tau_{\eta}$. Setting $\bar{u}(t)=u(t)$ for $t \in\left[0, s_{j_{0}}\right]$ and $\bar{u}(t)=\tilde{u}\left(t-s_{j_{0}}\right)$ for $t \in\left[s_{j_{0}}, s_{j_{0}}+T_{2}\right)$ we first note that $\bar{u}$ is a free energy solution to (1.3) on $\left[0, s_{j_{0}}+T_{2}\right)$ and a true extension of $u$ as 
$s_{j_{0}}+T_{2} \geq T_{1}-\left(\tau_{\eta} / 2\right)+\tau_{\eta} \geq T_{1}+\left(\tau_{\eta} / 2\right)$. We then iterate this construction as long as the alternative stated in Theorem 2.4 is not fulfilled to complete the proof.

Thanks to the regularity of weak solutions we may next proceed as in the proof of (2.8) to deduce (2.10).

Corollary 2.5 (Lower bound on the blow-up rate) Let u be the free energy solution to (1.3) on $\left[0, T_{\omega}\right)$ constructed in Theorem 2.4 with an initial condition $u_{0}$ satisfying (1.2). If $T_{\omega}$ is finite, then

$$
\|u(t)\|_{m} \geq\left[C_{0}\left(T_{\omega}-t\right)\right]^{-(m-1) / m},
$$

where $C_{0}$ is defined in Theorem 2.4.

Proof. Let $t \in\left(0, T_{\omega}\right)$ and $t_{2} \in\left(t, T_{\omega}\right)$. By (2.10), we have

$$
\left\|u\left(t_{2}\right)\right\|_{m}^{-m /(m-1)} \geq\|u(t)\|_{m}^{-m /(m-1)}-C_{0}\left(t_{2}-t\right) .
$$

Letting $t_{2}$ going to $T_{\omega}$ gives

$$
0 \geq\|u(t)\|_{m}^{-m /(m-1)}-C_{0}\left(T_{\omega}-t\right)
$$

hence the expected result.

Remark 2.6 No uniqueness result for (1.3) seems to be available in the literature. We thus stress that, from now on, all the results refer to the solutions constructed in Theorem 2.4.

\section{The free energy functional $\mathcal{F}$}

As we have just seen in the existence proof, the existence time of a free energy solution to (1.3) heavily depends on the behaviour of its $\mathrm{L}^{m}$-norm. As the free energy $\mathcal{F}$ involves the $\mathrm{L}^{m}$-norm, the information given $\mathcal{F}$ will be of paramount importance. Let us then proceed to a deeper study of this functional.

Lemma 3.1 (Scaling properties of the free energy) Given $h \in \mathrm{L}^{1}\left(\mathbb{R}^{d}\right) \cap \mathrm{L}^{m}\left(\mathbb{R}^{d}\right)$, let us define $h_{\lambda}(x):=\lambda^{d} h(\lambda x)$, then

$$
\mathcal{F}\left[h_{\lambda}\right]=\lambda^{d-2} \mathcal{F}[h] \quad \text { for all } \lambda \in(0, \infty) .
$$

Proof. We have

$$
\begin{aligned}
\mathcal{F}\left[h_{\lambda}\right] & =\frac{1}{m-1} \int_{\mathbb{R}^{d}} \lambda^{2 d-2} h(\lambda x)^{m} \mathrm{~d} x-\frac{c_{d}}{2} \iint_{\mathbb{R}^{d} \times \mathbb{R}^{d}} \lambda^{2 d} \frac{h(\lambda x) h(\lambda y)}{|x-y|^{d-2}} \mathrm{~d} x \mathrm{~d} y \\
& =\frac{\lambda^{d-2}}{m-1} \int_{\mathbb{R}^{d}} h(x)^{m} \mathrm{~d} x-\frac{c_{d} \lambda^{d-2}}{2} \iint_{\mathbb{R}^{d} \times \mathbb{R}^{d}} \frac{h(x) h(y)}{|x-y|^{d-2}} \mathrm{~d} x \mathrm{~d} y \\
& =\lambda^{d-2} \mathcal{F}[h],
\end{aligned}
$$

giving the announced scaling property.

We next establish a variant to the Hardy-Littlewood-Sobolev (VHLS) inequality:

Lemma 3.2 (VHLS inequality) For $h \in \mathrm{L}^{1}\left(\mathbb{R}^{d}\right) \cap \mathrm{L}^{m}\left(\mathbb{R}^{d}\right)$ we put

$$
\mathcal{W}(h):=\iint_{\mathbb{R}^{d} \times \mathbb{R}^{d}} \frac{h(x) h(y)}{|x-y|^{d-2}} \mathrm{~d} x \mathrm{~d} y .
$$

Then

$$
C_{*}:=\sup _{h \neq 0}\left\{\frac{\mathcal{W}(h)}{\|h\|_{1}^{2 / d}\|h\|_{m}^{m}}, h \in \mathrm{L}^{1}\left(\mathbb{R}^{d}\right) \cap \mathrm{L}^{m}\left(\mathbb{R}^{d}\right)\right\}<\infty .
$$


First recall the Hardy-Littlewood-Sobolev (HLS) inequality, see [25, Theorem 4.3], hich states that if

$$
\frac{1}{p}+\frac{1}{q}+\frac{\lambda}{d}=2 \text { and } 0<\lambda<d,
$$

then for all $f \in \mathrm{L}^{p}\left(\mathbb{R}^{d}\right), g \in \mathrm{L}^{q}\left(\mathbb{R}^{d}\right)$, there exists a sharp positive constant $C_{\mathrm{HLS}}>0$, given by [24], which only depends on $p, q$ and $\lambda$ such that

$$
\left|\iint_{\mathbb{R}^{d} \times \mathbb{R}^{d}} \frac{f(x) g(y)}{|x-y|^{\lambda}} \mathrm{d} x \mathrm{~d} y\right| \leq C_{\mathrm{HLS}}\|f\|_{p}\|g\|_{q} .
$$

Proof of Lemma 3.2. Consider $h \in \mathrm{L}^{1}\left(\mathbb{R}^{d}\right) \cap \mathrm{L}^{m}\left(\mathbb{R}^{d}\right)$. Applying the HLS inequality (3.2) with $p=q=2 d /(d+2)$ and $\lambda=d-2$, and then the Hölder inequality with $1<p=2 d /(d+2)<m$, we obtain

$$
|\mathcal{W}(h)|=\left|\iint_{\mathbb{R}^{d} \times \mathbb{R}^{d}} \frac{h(x) h(y)}{|x-y|^{d-2}} \mathrm{~d} x \mathrm{~d} y\right| \leq C_{\mathrm{HLS}}\|h\|_{p}^{2} \leq C_{\mathrm{HLS}}\|h\|_{1}^{2 / d}\|h\|_{m}^{m} .
$$

Consequently, $C_{*}$ is finite and bounded from above by $C_{\mathrm{HLS}}$.

We next turn to the existence of maximisers for the VHLS inequality which can be proved by similar arguments as for the classical HLS inequality in [24, Theorem 2.5].

Lemma 3.3 (Extremals of the VHLS inequality) There exists a non-negative, radially symmetric and non-increasing function $P_{*} \in \mathrm{L}^{1}\left(\mathbb{R}^{d}\right) \cap \mathrm{L}^{m}\left(\mathbb{R}^{d}\right)$ such that $\mathcal{W}\left(P_{*}\right)=C_{*}$ with $\left\|P_{*}\right\|_{1}=$ $\left\|P_{*}\right\|_{m}=1$.

Proof. Define

$$
\Lambda(h):=\frac{\mathcal{W}(h)}{\|h\|_{1}^{2 / d}\|h\|_{m}^{m}} \quad \text { for } h \in \mathrm{L}^{1}\left(\mathbb{R}^{d}\right) \cap \mathrm{L}^{m}\left(\mathbb{R}^{d}\right),
$$

and consider a maximising sequence $\left(p_{j}\right)_{j}$ in $\mathrm{L}^{1}\left(\mathbb{R}^{d}\right) \cap \mathrm{L}^{m}\left(\mathbb{R}^{d}\right)$, that is

$$
\lim _{j \rightarrow \infty} \Lambda\left(p_{j}\right)=C_{*} .
$$

Step 1 - We first prove that we may assume that $p_{j}$ is a non-negative, radially symmetric, non-increasing function such that $\left\|p_{j}\right\|_{1}=\left\|p_{j}\right\|_{m}=1$ for any $j \geq 0$. Indeed, $\Lambda\left(p_{j}\right) \leq \Lambda\left(\left|p_{j}\right|\right)$ so that $\left(\left|p_{j}\right|\right)_{j}$ is also a maximising sequence. Next, let us introduce $\tilde{p}_{j}(x):=\lambda_{j}\left|p_{j}\left(\mu_{j} x\right)\right|$ with $\mu_{j}:=$ $\left(\left\|p_{j}\right\|_{1} /\left\|p_{j}\right\|_{m}\right)^{m /[d(m-1)]}$ and $\lambda_{j}:=\mu_{j}^{d} /\left\|p_{j}\right\|_{1}$. A direct computation shows that $\Lambda\left(\tilde{p}_{j}\right)=\Lambda\left(\left|p_{j}\right|\right)$ and $\left\|\tilde{p}_{j}\right\|_{1}=\left\|\tilde{p}_{j}\right\|_{m}=1$. Finally, denoting by $p_{j}^{*}$ the symmetric decreasing rearrangement of $\tilde{p}_{j}$, we infer from the Riesz rearrangement properties [24, Lemma 2.1] that

$$
\Lambda\left(p_{j}^{*}\right)=\mathcal{W}\left(p_{j}^{*}\right) \geq \mathcal{W}\left(\tilde{p}_{j}\right)=\Lambda\left(\tilde{p}_{j}\right)=\Lambda\left(\left|p_{j}\right|\right) .
$$

Consequently, $\left(p_{j}^{*}\right)_{j}$ is also a maximising sequence and the first step is proved.

Step 2 - Let us now prove that the supremum is achieved. For $k \in\{1, m\}$, the monotonicity and the non-negativity of $p_{j}$ imply that

$$
1=\left\|p_{j}\right\|_{k}^{k}=d|B(0,1)| \int_{0}^{\infty} r^{d-1} p_{j}^{k}(r) \mathrm{d} r \geq d|B(0,1)| p_{j}^{k}(R) \int_{0}^{R} r^{d-1} \mathrm{~d} r \geq|B(0,1)| R^{d} p_{j}^{k}(R) .
$$

So that

$$
0 \leq p_{j}(R) \leq b(R):=C_{1} \inf \left\{R^{-d / m} ; R^{-d}\right\} \quad \text { for } R>0
$$


Now, we use once more the monotonicity of the $p_{j}$ 's and their boundedness in $(R, \infty)$ for any $R>0$ to deduce from Helly's theorem that there are a sub-sequence of $\left(p_{j}\right)_{j}$ (not relabelled) and a non-negative and non-increasing function $P_{*}$ such that $\left(p_{j}\right)_{j}$ converges to $P_{*}$ point-wisely. In addition, as $1<2 d /(d+2)<m, x \mapsto b(|x|)$ belongs to $\mathrm{L}^{2 d /(d+2)}\left(\mathbb{R}^{d}\right)$ while the HLS inequality (3.2) warrants that

$$
(x, y) \mapsto b(|x|) b(|y|)|x-y|^{-(d-2)} \in \mathrm{L}^{1}\left(\mathbb{R}^{d} \times \mathbb{R}^{d}\right) .
$$

Together with (3.4) and the point-wise convergence of $\left(p_{j}\right)_{j}$, this implies that

$$
\lim _{j \rightarrow \infty} \mathcal{W}\left(p_{j}\right)=\mathcal{W}\left(P_{*}\right)
$$

by the Lebesgue dominated convergence theorem. Consequently, $\mathcal{W}\left(P_{*}\right)=C_{*}$ and thus $P_{*} \neq 0$. In addition, the point-wise convergence of $\left(p_{j}\right)_{j}$ and Fatou's lemma ensure $\left\|P_{*}\right\|_{1} \leq 1$ and $\left\|P_{*}\right\|_{m} \leq 1$. Therefore $\Lambda\left(P_{*}\right) \geq C_{*}$ and using (3.3) we conclude that $\Lambda\left(P_{*}\right)=C_{*}$. This in turn implies that $\left\|P_{*}\right\|_{1}=\left\|P_{*}\right\|_{m}=1$.

We are now in a position to begin the study of the free energy functional $\mathcal{F}$. To this end, let us define the critical mass $M_{c}$ by

$$
M_{c}:=\left[\frac{2}{(m-1) C_{*} c_{d}}\right]^{d / 2} .
$$

Next, for $M>0$, we put

$$
\mu_{M}:=\inf _{h \in \mathcal{Y}_{M}} \mathcal{F}[h] \quad \text { where } \quad \mathcal{Y}_{M}:=\left\{h \in \mathrm{L}^{1}\left(\mathbb{R}^{d}\right) \cap \mathrm{L}^{m}\left(\mathbb{R}^{d}\right):\|h\|_{1}=M\right\},
$$

and first identify the values of $\mu_{M}$ as a function of $M>0$.

Proposition 3.4 (Infimum of the free energy) We have

$$
\mu_{M}= \begin{cases}0 & \text { if } M \in\left(0, M_{c}\right], \\ -\infty & \text { if } M>M_{c} .\end{cases}
$$

Moreover,

$$
\frac{C_{*} c_{d}}{2}\left(M_{c}^{2 / d}-M^{2 / d}\right)\|h\|_{m}^{m} \leq \mathcal{F}[h] \leq \frac{C_{*} c_{d}}{2}\left(M_{c}^{2 / d}+M^{2 / d}\right)\|h\|_{m}^{m}
$$

for $h \in \mathcal{Y}_{M}$. Furthermore, the infimum $\mu_{M}$ is not achieved if $M<M_{c}$ while there exists one minimiser of $\mathcal{F}$ in $\mathcal{Y}_{M_{c}}$.

Proof. Consider $h \in \mathrm{L}^{1}\left(\mathbb{R}^{d}\right) \cap \mathrm{L}^{m}\left(\mathbb{R}^{d}\right)$. By the VHLS inequality (3.1),

$$
\mathcal{F}[h] \geq\left(\frac{1}{m-1}-\frac{C_{*} c_{d}}{2} M^{2 / d}\right)\|h\|_{m}^{m} \geq \frac{C_{*} c_{d}}{2}\left(M_{c}^{2 / d}-M^{2 / d}\right)\|h\|_{m}^{m},
$$

and

$$
\mathcal{F}[h] \leq\left(\frac{1}{m-1}+\frac{C_{*} c_{d}}{2} M^{2 / d}\right)\|h\|_{m}^{m} \leq \frac{C_{*} c_{d}}{2}\left(M_{c}^{2 / d}+M^{2 / d}\right)\|h\|_{m}^{m},
$$

hence (3.7).

Case $M \leq M_{c}$ - By (3.7), $\mathcal{F}$ is non-negative, so that $\mu_{M} \geq 0$. Choosing

$$
h_{*}(t, x)=\frac{M}{(2 \pi t)^{d / 2}} \mathrm{e}^{-|x|^{2} /(4 t)}
$$


then

$$
\left\|h_{*}(t)\right\|_{1}=M \quad \text { and } \quad\left\|h_{*}(t)\right\|_{m}^{m}=O\left(t^{-d(m-1) / 2}\right) .
$$

Therefore $h_{*}(t)$ belongs to $\mathcal{Y}_{M}$ for each $t>0$ and it follows from $(3.7)$ that $\mathcal{F}\left[h_{*}(t)\right] \rightarrow 0$ as $t \rightarrow \infty$. The infimum $\mu_{M}$ of $\mathcal{F}$ on $\mathcal{Y}_{M}$ is thus non-positive, hence $\mu_{M}=0$.

Finally, in the case $M<M_{c}, \mu_{M}=0$ and (3.7) imply that the infimum of $\mathcal{F}$ in $\mathcal{Y}_{M}$ is not achieved. If $M=M_{c}$ and $p \in \mathrm{L}^{1}\left(\mathbb{R}^{d}\right) \cap \mathrm{L}^{m}\left(\mathbb{R}^{d}\right.$ ) satisfies $\mathcal{W}(p)=C_{*}\|p\|_{m}^{m}\|p\|_{1}^{2 / d}$ (such a function exists by Lemma 3.3), then

$$
\tilde{p}(x):=M_{c}^{-d /(d-2)} p\left(x M_{c}^{-m /(d-2)}\right)
$$

belongs to $\mathcal{Y}_{M_{c}}$ with $\|\tilde{p}\|_{m}=1$ and $\mathcal{W}(\tilde{p})=C_{*} M_{c}^{2 / d}$. Therefore, $\mathcal{F}[\tilde{p}]=0$ and we have thus proved that suitably rescaled extremals of the VHLS inequality (3.1) are minimisers for $\mathcal{F}$ in $\mathcal{Y}_{M_{c}}$.

Case $M>M_{c}$ - This part of the proof is based on arguments in [39]. Fix $\theta \in\left(\left(M_{c} / M\right)^{2 / d}, 1\right)$. By the VHLS inequality (3.1), there exists a non-zero function $h^{*} \in \mathrm{L}^{1}\left(\mathbb{R}^{d}\right) \cap \mathrm{L}^{m}\left(\mathbb{R}^{d}\right)$, such that

$$
\theta C_{*} \leq \frac{\left|\mathcal{W}\left(h^{*}\right)\right|}{\left\|h^{*}\right\|_{m}^{m}\left\|h^{*}\right\|_{1}^{2 / d}} \leq C_{*}
$$

Since $\left|\mathcal{W}\left(h^{*}\right)\right| \leq \mathcal{W}\left(\left|h^{*}\right|\right)$ we may assume without loss of generality that $h^{*}$ is non-negative. Let $\lambda>0$ and consider the function $h_{\lambda}(x):=\lambda^{d} h^{*}\left(\lambda\left\|h^{*}\right\|_{1}^{1 / d} M^{-1 / d} x\right)$. Then, $h_{\lambda} \in \mathcal{Y}_{M}$ and it follows from the definition of $M_{c}$ and (3.8) that

$$
\begin{aligned}
\mathcal{F}\left[h_{\lambda}\right] & =\lambda^{d-2}\left[\frac{M}{(m-1)\left\|h^{*}\right\|_{1}}\left\|h^{*}\right\|_{m}^{m}-\frac{c_{d}}{2}\left(\frac{M}{\left\|h^{*}\right\|_{1}}\right)^{(d+2) / d} \mathcal{W}\left(h^{*}\right)\right] \\
& \leq \lambda^{d-2}\left[\frac{M}{(m-1)\left\|h^{*}\right\|_{1}}\left\|h^{*}\right\|_{m}^{m}-\frac{c_{d}}{2}\left(\frac{M}{\left\|h^{*}\right\|_{1}}\right)^{(d+2) / d} \theta C_{*}\left\|h^{*}\right\|_{m}^{m}\left\|h^{*}\right\|_{1}^{2 / d}\right] \\
& =\lambda^{d-2}\left(\frac{M}{\left\|h^{*}\right\|_{1}}\right)^{(d+2) / d} \frac{\left\|h^{*}\right\|_{m}^{m}}{m-1}\left[\left(\frac{M_{c}}{M}\right)^{2 / d}-\theta\right] .
\end{aligned}
$$

Owing to the choice of $\theta$ we may let $\lambda$ go to infinity to obtain that $\mu_{M}=-\infty$, thus completing the proof.

Let us now describe the set of minimisers of $\mathcal{F}$ in $\mathcal{Y}_{M_{c}}$.

Proposition 3.5 (Identification of the minimisers) Let $\zeta$ be the unique positive radial classical solution to

$$
\Delta \zeta+\frac{m-1}{m} \zeta^{1 /(m-1)}=0 \quad \text { in } \quad B(0,1) \text { with } \zeta=0 \quad \text { on } \quad \partial B(0,1) .
$$

If $V$ is a minimiser of $\mathcal{F}$ in $\mathcal{Y}_{M_{c}}$ there are $R>0$ and $z \in \mathbb{R}^{d}$ such that

$$
V(x)= \begin{cases}\frac{1}{R^{d}}\left[\zeta\left(\frac{x-z}{R}\right)\right]^{d /(d-2)} & \text { if } x \in B(z, R), \\ 0 & \text { if } x \in \mathbb{R}^{d} \backslash B(z, R) .\end{cases}
$$


Proof. We have already shown in Proposition 3.4 that the function $\mathcal{F}$ has at least a minimiser in $\mathcal{Y}_{M_{c}}$. Let $V$ be a minimiser of $\mathcal{F}$ in $\mathcal{Y}_{M_{c}}$, and define $\tilde{V}(x):=\|V\|_{m}^{-m /(m-1)} V\left(x\|V\|_{m}^{-m /(d(m-1))}\right)$ for $x \in \mathbb{R}^{d}$. We have $\|\tilde{V}\|_{1}=M_{c},\|\tilde{V}\|_{m}=1$ and $\mathcal{F}[\tilde{V}]=0$, so that $\tilde{V}$ is also a minimiser of $\mathcal{F}$ in $\mathcal{Y}_{M_{c}}$. We next denote by $W$ the symmetric rearrangement of $\tilde{V}$. Then $\|W\|_{1}=M_{c}$, $\|W\|_{m}=\|\tilde{V}\|_{m}=1$ and $\mathcal{W}(W) \geq|\mathcal{W}(\tilde{V})|$ by the Riesz rearrangement properties [24, Lemma 2.1]. Therefore, $\mathcal{F}[W] \leq \mathcal{F}[\tilde{V}]=0$ and thus $\mathcal{F}[W]=0$ since $W \in \mathcal{Y}_{M_{c}}$. This in turn implies that $\mathcal{W}(W)=|\mathcal{W}(\tilde{V})|$. Again by $\left[24\right.$, Lemma 2.1] there is $y \in \mathbb{R}^{d}$ such that $\tilde{V}(x)=W(x-y)$ for $y \in \mathbb{R}^{d}$.

We next derive the Euler-Lagrange equation solved by $W$ and first point out that a difficulty arises from the non-differentiability of the $\mathrm{L}^{1}$-norm. Nevertheless, we introduce $\Sigma_{0}:=\left\{x \in \mathbb{R}^{d}\right.$ : $W(x)=0\}, \Sigma_{+}:=\left\{x \in \mathbb{R}^{d}: W(x)>0\right\}$ and consider $\varphi \in \mathcal{C}_{0}^{\infty}\left(\mathbb{R}^{d}\right)$ and $\varepsilon>0$. The perturbation $M_{c}\|W+\varepsilon \varphi\|_{1}^{-1}(W+\varepsilon \varphi)$ belongs to $\mathcal{Y}_{M_{c}}$ and is such that

$$
\mathcal{F}\left[\frac{M_{c}}{\|W+\varepsilon \varphi\|_{1}}(W+\varepsilon \varphi)\right] \geq \mathcal{F}[W] \geq 0 .
$$

After a few computations that we omit here we may let $\varepsilon \rightarrow 0$, and conclude that

$$
\begin{aligned}
& 2 \iint_{\mathbb{R}^{d} \times \mathbb{R}^{d}} \frac{W(x) \varphi(y)}{|x-y|^{d-2}} \mathrm{~d} y \mathrm{~d} x \\
& \quad \leq C_{*} M_{c}^{2 / d} m \int_{\mathbb{R}^{d}} W^{m-1}(x) \varphi(x) \mathrm{d} x+\frac{2}{d} C_{*} M_{c}^{(2-d) / d}\left(\int_{\Sigma_{+}} \varphi(x) \mathrm{d} x+\int_{\Sigma_{0}}|\varphi(x)| \mathrm{d} x\right) .
\end{aligned}
$$

Using the definition of $M_{c}$ and $\mathcal{K}$, the above formula also reads

$$
\int_{\mathbb{R}^{d}}\left(\frac{m}{m-1} W^{m-1}-\mathcal{K} * W+\frac{2-m}{m-1} \frac{1}{M_{c}}\right) \varphi \mathrm{d} x \geq \frac{2-m}{m-1} \frac{1}{M_{c}} \int_{\Sigma_{0}}(\varphi-|\varphi|) \mathrm{d} x
$$

for all $\varphi \in \mathcal{C}_{0}^{\infty}\left(\mathbb{R}^{d}\right)$. On the one hand, the right-hand side of (3.9) vanishes for any non-negative $\varphi \in \mathcal{C}_{0}^{\infty}\left(\mathbb{R}^{d}\right)$, so that

$$
\frac{m}{m-1} W^{m-1}-\mathcal{K} * W+\frac{2-m}{m-1} \frac{1}{M_{c}} \geq 0 \quad \text { a.e. in } \quad \mathbb{R}^{d} .
$$

Therefore, for almost every $x \in \Sigma_{0}$, we have $0 \geq \mathcal{K} * W(x)-(2-m) /\left[(m-1) M_{c}\right]$ so that

$$
\frac{m}{m-1} W^{m-1}(x)=0=\left(\mathcal{K} * W(x)-\frac{2-m}{m-1} \frac{1}{M_{c}}\right)_{+} \quad \text { for almost every } \quad x \in \Sigma_{0} .
$$

On the other hand, if $\psi \in \mathcal{C}_{0}^{\infty}\left(\mathbb{R}^{d}\right)$, a standard approximation argument allows us to take $\varphi=$ $\mathbf{1}_{\Sigma_{+}} \psi$ in (3.9) and deduce that

$$
\int_{\Sigma_{+}}\left(\frac{m}{m-1} W^{m-1}-\mathcal{K} * W+\frac{2-m}{m-1} \frac{1}{M_{c}}\right) \psi \mathrm{d} x \geq 0 .
$$

This inequality being also valid for $-\psi$, we conclude that the left-hand side of the above inequality vanishes for all $\psi \in \mathcal{C}_{0}^{\infty}\left(\mathbb{R}^{d}\right)$, whence

$$
\frac{m}{m-1} W^{m-1}=\mathcal{K} * W-\frac{2-m}{m-1} \frac{1}{M_{c}} \text { a.e. in } \quad \Sigma_{+} .
$$

Combining (3.10) and (3.11) gives

$$
\frac{m}{m-1} W^{m-1}=\left(\mathcal{K} * W-\frac{2-m}{m-1} \frac{1}{M_{c}}\right)_{+} \text {a.e. in } \mathbb{R}^{d} .
$$


Now, since $W$ is radially symmetric and non-increasing there exists $\rho \in(0, \infty]$ such that

$$
\Sigma_{+} \subset B(0, \rho) \text { and } \Sigma_{0} \subset \mathbb{R}^{d} \backslash B(0, \rho),
$$

and we infer from (3.11) that

$$
\frac{m}{m-1} W^{m-1}=\mathcal{K} * W-\frac{2-m}{m-1} \frac{1}{M_{c}} \quad \text { for a.e. } x \in B(0, \rho) .
$$

Since $W \in \mathrm{L}^{r}\left(\mathbb{R}^{d}\right)$ for each $r \in(1, m]$ it follows from the HLS inequality (3.2) that $\mathcal{K} * W \in \mathrm{L}^{r}\left(\mathbb{R}^{d}\right)$ for each $r \in\left(d /(d-2), m /(m-1)^{2}\right]$, see [25, Theorem 10.2]. In particular, $\mathcal{K} * W$ and $W^{m-1}$ both belong to $L^{m /(m-1)}\left(\mathbb{R}^{d}\right)$. This property and (3.12) then exclude that $\rho=\infty$ as $M_{c}>0$. Therefore $\rho<\infty$ and

$$
\frac{m}{m-1} W^{m-1}(x)= \begin{cases}\mathcal{K} * W(x)-\frac{2-m}{m-1} \frac{1}{M_{c}} & \text { if }|x|<\rho, \\ 0 & \text { if }|x|>\rho .\end{cases}
$$

Since $\mathcal{K} * W \in \mathrm{L}^{m /(m-1)^{2}}\left(\mathbb{R}^{d}\right)$, the above inequality allows us to conclude that $W \in \mathrm{L}^{m /(m-1)}\left(\mathbb{R}^{d}\right)$. We now improve the regularity of $W$ by classical elliptic estimates. Introduce $\theta:=W^{m-1}$ and note that

$$
\frac{m}{m-1} \theta(x)=\int_{\mathbb{R}^{d}} \mathcal{K}(x-y) W(y) \mathrm{d} y+\frac{m-1}{m-2} \frac{1}{M_{c}}
$$

for $x \in B(0, \rho)$ and $W \in \mathrm{L}^{m /(m-1)}\left(\mathbb{R}^{d}\right)$. By [19, Theorem 9.9], we have $\theta \in W^{2, m /(m-1)}(B(0, \rho))$. A bootstrap argument then ensures that $\theta$ and $W$ both belong to $W^{2, r}(B(0, \rho))$ for every $r \in$ $(1, \infty)$. It then follows from [19, Lemma 4.2] that $\theta \in \mathcal{C}^{2}(B(0, \rho))$ with $-\Delta \theta=(m-1) \theta^{m /(m-1)} / m$ in $B(0, \rho)$ while $\left[19\right.$, Lemma 4.1] warrants that $\theta \in \mathcal{C}^{1}\left(\mathbb{R}^{d}\right)$. Then $\theta(x)=0$ if $|x|=\rho$ and $\theta$ is thus a classical solution to $-\Delta \theta=(m-1) \theta^{m /(m-1)} / m$ in $B(0, \rho)$ with $\theta=0$ on $\partial B(0, \rho)$. By $[18$, Lemma 2.3], there is a unique positive solution to this problem. In fact, a simple scaling argument shows that

$$
\theta(x)=\rho^{2(m-1) /(m-2)} \zeta\left(\frac{x}{\rho}\right) \quad \text { for } x \text { in } B(0, \rho)
$$

and then

$$
W(x)=\frac{1}{\rho^{d}}\left[\zeta\left(\frac{x}{\rho}\right)\right]^{d /(d-2)} \quad \text { for } x \text { in } B(0, \rho) .
$$

Coming back to $V$, we have

$$
V(x)= \begin{cases}\lambda^{d} W(\lambda x-y)=0 & \text { if } x \in \mathbb{R}^{d} \backslash B\left(\frac{y}{\lambda}, \frac{\rho}{\lambda}\right), \\ \left(\frac{\lambda}{\rho}\right)^{d}\left[\zeta\left(\left(x-\frac{y}{\lambda}\right)\left(\frac{\rho}{\lambda}\right)^{-1}\right)\right]^{d /(d-2)} & \text { if } x \in B\left(\frac{y}{\lambda}, \frac{\rho}{\lambda}\right),\end{cases}
$$

which is the desired result with $R=\rho / \lambda$ and $z=y / \lambda$.

Remark 3.6 As a consequence of the identification of the minimisers given in Proposition 3.5, $C_{*}<C_{\mathrm{HLS}}$. Otherwise any minimiser $V$ of $\mathcal{F}$ is $\mathcal{Y}_{M_{c}}$ would also be an extremum for the $H L S$ inequality (3.2) and thus be equal to

$$
V(x)=\frac{a}{\left(1+|x|^{2}\right)^{(d+2) / 2}},
$$

for some $a>0$, see [24, Theorem 3.1]. This contradicts Proposition 3.5. 
Lemma 3.7 (Unboundedness of $\mathcal{F}$ ) For each $M>0$ we have

$$
\sup _{h \in \mathcal{Y}_{M}} \mathcal{F}[h]=+\infty .
$$

If $M \in\left(0, M_{c}\right)$ the claim (3.13) is actually a straightforward consequence of (3.7).

Proof. Let $M>0$ and assume for contradiction that

$$
A:=\sup _{h \in \mathcal{Y}_{M}} \mathcal{F}[h]<\infty
$$

Consider $h \in \mathrm{L}^{1}\left(\mathbb{R}^{d}\right) \cap \mathrm{L}^{m}\left(\mathbb{R}^{d}\right)$ and define $h_{\lambda}(x):=M \lambda^{d} h(\lambda x) /\|h\|_{1}$ for $x \in \mathbb{R}^{d}$ and $\lambda>0$. Then $\left\|h_{\lambda}\right\|_{1}=M$ so that $h_{\lambda} \in \mathcal{Y}_{M}$ with

$$
\left\|h_{\lambda}\right\|_{m}^{m}=\lambda^{d-2}\left(\frac{M}{\|h\|_{1}}\right)^{m}\|h\|_{m}^{m} \text { and } \mathcal{W}\left(h_{\lambda}\right)=\lambda^{d-2}\left(\frac{M}{\|h\|_{1}}\right)^{2} \mathcal{W}(h) .
$$

Since $h_{\lambda} \in \mathcal{Y}_{M}$ we have $\mathcal{F}\left[h_{\lambda}\right] \leq A$, hence

$$
\left\|h_{\lambda}\right\|_{m}^{m} \leq(m-1)\left(A+\frac{c_{d}}{2} \mathcal{W}\left(h_{\lambda}\right)\right)
$$

from which we deduce

$$
\begin{aligned}
\|h\|_{m}^{m} & \leq(m-1)\left(A \lambda^{2-d}\left(\frac{\|h\|_{1}}{M}\right)^{m}+\frac{c_{d}}{2}\left(\frac{M}{\|h\|_{1}}\right)^{2 / d} \mathcal{W}(h)\right) \\
& \leq(m-1) A \lambda^{2-d}\left(\frac{\|h\|_{1}}{M}\right)^{m}+\frac{1}{C_{*} M_{c}^{2 / d}}\left(\frac{M}{\|h\|_{1}}\right)^{2 / d} \mathcal{W}(h) .
\end{aligned}
$$

This inequality being valid for all $\lambda>0$ we let $\lambda \rightarrow \infty$ and use the HLS inequality (3.2) to obtain

$$
\|h\|_{m}^{m} \leq \frac{1}{C_{*} M_{c}^{2 / d}}\left(\frac{M}{\|h\|_{1}}\right)^{2 / d} \mathcal{W}(h) \leq\left(\frac{M}{M_{c}}\right)^{2 / d} \frac{C_{\mathrm{HLS}}}{C_{*}} \frac{\|h\|_{2 d /(d+2)}^{2}}{\|h\|_{1}^{2 / d}} .
$$

Consequently,

$$
\|h\|_{m}^{m}\|h\|_{1}^{2 / d} \leq\left(\frac{M}{M_{c}}\right)^{2 / d} \frac{C_{\mathrm{HLS}}}{C_{*}}\|h\|_{2 d /(d+2)}^{2}
$$

for all $h \in \mathrm{L}^{1}\left(\mathbb{R}^{d}\right) \cap \mathrm{L}^{m}\left(\mathbb{R}^{d}\right)$.

Now, as $2 d /(d+2)<m$, we may choose $\gamma \in((d+2) / d, d / m)$ and put $b_{\delta}(x):=(\delta+$ $|x|)^{-\gamma} \mathbb{1}_{B(0,1)}(x)$ for $x \in \mathbb{R}^{d}$ and $\delta \in[0,1]$. Clearly $b_{\delta}$ belongs to $\mathrm{L}^{1}\left(\mathbb{R}^{d}\right) \cap \mathrm{L}^{m}\left(\mathbb{R}^{d}\right)$ with $\left\|b_{\delta}\right\|_{1} \geq$ $\left\|b_{1}\right\|_{1}>0$ and $\left\|b_{\delta}\right\|_{2 d /(d+2)} \leq\left\|b_{0}\right\|_{2 d /(d+2)}<\infty$ for each $\delta \in(0,1]$. These properties and (3.14) readily imply that $\left(b_{\delta}\right)_{\delta \in(0,1]}$ is bounded in $\mathrm{L}^{m}\left(\mathbb{R}^{d}\right)$ which is clearly not true according to the choice of $\gamma$. Therefore $A$ cannot be finite and Lemma 3.7 is proved.

\section{Critical threshold}

It turns out that the critical mass $M_{c}$ arising in the study of the free energy functional and defined in (3.5) plays also an important role in the dynamics of (1.3). In the next sections we will distinguish the three cases $M>M_{c}$ (super-critical case), $M<M_{c}$ (sub-critical case), and $M=M_{c}$ (critical case), $M$ denoting the $L^{1}\left(\mathbb{R}^{d}\right)$-norm of the initial condition $u_{0}$. 


\subsection{Finite time blow-up in the super-critical case}

We start with the case $M>M_{c}$ in which we use the standard argument relying on the evolution of the second moment of solutions as originally done in [21] for the PKS system corresponding to $d=2$ and $m=1$.

Lemma 4.1 (Virial identity) Under assumption (1.2), let u be a free energy solution to (1.3) on $[0, T)$ with initial condition $u_{0}$ for some $T \in(0, \infty]$. Then

$$
\frac{\mathrm{d}}{\mathrm{d} t} \int_{\mathbb{R}^{d}}|x|^{2} u(t, x) \mathrm{d} x=2(d-2) \mathcal{F}[u(t)], \quad t \in[0, T) .
$$

Proof. Here, we show the formal computation leading to this property, the passing to the limit from the approximated problem (2.1) can be done by adapting the arguments in [34, Lemma 6.2] and [7, Lemma 2.1] without any further complication. By integration by parts in (1.3) and symmetrising the second term, we obtain

$$
\begin{aligned}
\frac{\mathrm{d}}{\mathrm{d} t} \int_{\mathbb{R}^{d}}|x|^{2} u(t, x) \mathrm{d} x & =2 d \int_{\mathbb{R}^{d}} u^{m}(t, x) \mathrm{d} x+2 \iint_{\mathbb{R}^{d} \times \mathbb{R}^{d}}[x \cdot \nabla \mathcal{K}(x-y)] u(t, x) u(t, y) \mathrm{d} y \mathrm{~d} x \\
& =2 d \int_{\mathbb{R}^{d}} u^{m}(t, x) \mathrm{d} x+\iint_{\mathbb{R}^{d} \times \mathbb{R}^{d}}[(x-y) \cdot \nabla \mathcal{K}(x-y)] u(t, x) u(t, y) \mathrm{d} y \mathrm{~d} x \\
& =2(d-2) \mathcal{F}[u(t)],
\end{aligned}
$$

giving the desired identity.

Remark 4.2 Let us mention that a similar argument can be found in [34, Lemma 6.2] and [35] in the present situation where the Poisson equation is substituted by $-\Delta \phi=u-\phi$. The previous evolution for the second moment is simpler in our case than the one in [35] and resembles that arising in the study of critical non-linear Schrödinger equations [41, 14]. Actually, for the NLS equation the virial identity involves the second time derivative of the second moment which is shown to be bounded from above by a concave function vabishing at some finite time. Let us stress that, in our case, the second moment is always concave in time since its second time derivative is given by the dissipation of the free-energy functional, see (1.4).

Let us also emphasise that this second moment evolution is more complicated than in the classical PKS system corresponding to $d=2$ and $m=1$ where the time derivative of the second moment is a constant.

An easy consequence of the previous lemma is the following blow-up result.

Proposition 4.3 (Blowing-up solutions) If $M>M_{c}$, then there are initial data $u_{0}$ satisfying (1.2) with $\left\|u_{0}\right\|_{1}=M$ and negative free energy $\mathcal{F}\left[u_{0}\right]$. Moreover, if $u_{0}$ is such an initial condition and $u$ denotes a free energy solution to $(1.3)$ on $\left[0, T_{\omega}\right)$ with initial condition $u_{0}$, then $T_{\omega}<\infty$ and the $\mathrm{L}^{m}$-norm of $u$ blows up in finite time.

Proof. The proof is based on the idea of Weinstein [39]. By the identification of the minimisers for the critical mass given in Proposition 3.5, $\tilde{u}:=\zeta^{d /(d-2)}$ satisfies (1.2) as well as $\|\tilde{u}\|_{1}=M_{c}$ and $\mathcal{F}[\tilde{u}]=0$. For $M>M_{c}$, the initial condition $u_{0}=\left(M / M_{c}\right) \tilde{u}$ also satisfies (1.2) with $\left\|u_{0}\right\|_{1}=M$ and

$$
\begin{aligned}
\mathcal{F}\left[u_{0}\right] & =\frac{1}{m-1}\left(\frac{M}{M_{c}}\right)^{m}\|\tilde{u}\|_{m}^{m}-\left(\frac{M}{M_{c}}\right)^{2} \frac{c_{d}}{2} \mathcal{W}(\tilde{u}) \\
& =\frac{1}{m-1}\left[\left(\frac{M}{M_{c}}\right)^{m}-\left(\frac{M}{M_{c}}\right)^{2}\right]\|\tilde{u}\|_{m}^{m},
\end{aligned}
$$


is negative as $M>M_{c}$ and $m<2$.

Consider next an initial condition $u_{0}$ satisfying (1.2) as well as $\left\|u_{0}\right\|_{1}>M_{c}$ and $\mathcal{F}\left[u_{0}\right]<0$. Denoting by $u$ a corresponding free energy solution to $(1.3)$ on $[0, T)$, we infer from the time monotonicity of $\mathcal{F}$ and Lemma 4.1 that

$$
\frac{\mathrm{d}}{\mathrm{d} t} \int_{\mathbb{R}^{d}}|x|^{2} u(t, x) \mathrm{d} x=2(d-2) \mathcal{F}[u(t)] \leq 2(d-2) \mathcal{F}\left[u_{0}\right]<0 .
$$

This implies that the second moment of $u(t)$ will become negative after some time and contradicts the non-negativity of $u$. Therefore, $T_{\omega}$ is finite and $\|u(t)\|_{m}$ blows up in finite time.

\subsection{Global existence}

Proposition 4.4 (Global existence in the subcritical case) Under assumption (1.2), there exists a free energy solution to $(1.3)$ in $[0, \infty)$ with initial condition $u_{0}$.

Proof. By Theorem 2.4 there are $T_{\omega}$ and a free energy solution to $(1.3)$ in $\left[0, T_{\omega}\right)$ with initial condition $u_{0}$. We then infer from (1.6), (1.7), and (3.7) that $u(t)$ belongs to $\mathcal{Y}_{M}$ for all $t \in\left[0, T_{\omega}\right)$ and

$$
\frac{C_{*} c_{d}}{2}\left(M_{c}^{2 / d}-M^{2 / d}\right)\|u(t)\|_{m}^{m} \leq \mathcal{F}[u(t)] \leq \mathcal{F}\left[u_{0}\right] .
$$

As $M<M_{c}$, we deduce from the previous inequality that $u$ lies in $\mathrm{L}^{\infty}\left(0, \min \left\{T, T_{\omega}\right\} ; \mathrm{L}^{m}\left(\mathbb{R}^{d}\right)\right)$ for every $T>0$ which implies that $T_{\omega}=\infty$ by Theorem 2.4 .

Let us now discuss the critical case.

\subsubsection{How would it blow-up?}

Proposition 4.5 (Nature of the blow-up) Let $u_{0}$ be an initial condition satisfying (1.2) with $\left\|u_{0}\right\|_{1}=M_{c}$ and consider a free energy solution $u$ to $(1.3)$ on $\left[0, T_{\omega}\right)$ with initial condition $u_{0}$ and $T_{\omega} \in(0, \infty]$ and such that $\|u(t)\|_{m} \rightarrow \infty$ as $t \nearrow T_{\omega}$. If $\left(t_{k}\right)_{k}$ is a sequence of positive real numbers such that $t_{k} \rightarrow T_{\omega}$ as $k \rightarrow \infty$, there are a sub-sequence $\left(t_{k_{j}}\right)_{j}$ of $\left(t_{k}\right)_{k}$ and a sequence $\left(x_{j}\right)_{j}$ in $\mathbb{R}^{d}$ such that

$$
\lim _{j \rightarrow \infty} \int_{\mathbb{R}^{d}}\left|u\left(t_{k_{j}}, x+x_{j}\right)-\frac{1}{\lambda_{k_{j}}^{d}} V\left(\frac{x}{\lambda_{k_{j}}}\right)\right| \mathrm{d} x=0,
$$

where $\lambda_{k}:=\left\|u\left(t_{k}\right)\right\|_{m}^{-m /(d-2)}$ and $V$ is the unique radially symmetric minimiser of $\mathcal{F}$ in $\mathcal{Y}_{M_{c}}$ such that $\|V\|_{m}=1$, see Proposition 3.5. Assume further that

$$
\mathcal{M}_{2}:=\sup _{t \in[0, T)} \int_{\mathbb{R}^{d}}|x|^{2} u(t, x) \mathrm{d} x<\infty,
$$

then

$$
\lim _{j \rightarrow \infty} x_{j}=\bar{x} \quad \text { where } \quad \bar{x}:=\frac{1}{M_{c}} \int_{\mathbb{R}^{d}} x u_{0}(x) \mathrm{d} x .
$$

Since $\mu_{M_{1}+M_{2}}=\mu_{M_{1}}+\mu_{M_{2}}$ for $M_{1} \leq M_{c}$ and $M_{2} \leq M_{c}$, the concentration compactness result as stated by P.-L. Lions [27] does not seem to apply directly. However, we follow the approach of M. Weinstein [40] to prove that the conclusion still holds true.

Proof. We set $v_{k}(x):=\lambda_{k}^{d} u\left(t_{k}, \lambda_{k} x\right)$ and aim at proving that $\left(v_{k}\right)_{k}$ converges strongly in $\mathrm{L}^{1}\left(\mathbb{R}^{d}\right)$. For this purpose we employ in Step 1 the concentration-compactness principle [27, Theorem II.1] 
to show that $\left(v_{k}\right)_{k}$ is tight up to translations. We argue in Step 2 as in [40, Theorem 1] to establish that $\left(v_{k}\right)_{k}$ has a limit in $\mathrm{L}^{1}\left(\mathbb{R}^{d}\right)$ and identify the limit. In the last step we use the additional bound on the second moment to show that the dynamics does not escape at infinity.

Step 1 - Tightness. Obviously,

$$
\left\|v_{k}\right\|_{1}=M_{c} \quad \text { and } \quad\left\|v_{k}\right\|_{m}=1 \quad \text { for } \quad k \geq 1
$$

The concentration-compactness principle [27] implies that there exists a sub-sequence (not relabelled) satisfying one of the three following properties:

(Compactness) There exists a sequence $\left(a_{k}\right)_{k}$ in $\mathbb{R}^{d}$ such that $\left(v_{k}\left(\cdot+a_{k}\right)\right)_{k} \in \mathbb{R}^{d}$ is tight, that is, for each $\varepsilon>0$ there is $R_{\varepsilon}>0$ such that

$$
\int_{B\left(a_{k}, R_{\varepsilon}\right)} v_{k}(x) \mathrm{d} x \geq M_{c}-\varepsilon
$$

(Vanishing) For all $R \geq 0$

$$
\lim _{k \rightarrow \infty} \sup _{y \in \mathbb{R}^{d}} \int_{B(y, R)} v_{k}(x) \mathrm{d} x=0
$$

(Dichotomy) There exists $\mu \in\left(0, M_{c}\right)$ such that for all $\varepsilon>0$, there exist $k_{0} \geq 1$ and three sequences of non-negative, integrable and compactly supported functions $\left(y_{k}^{\varepsilon}\right)_{k},\left(z_{k}^{\varepsilon}\right)_{k}$, and $\left(w_{k}^{\varepsilon}\right)_{k}$ satisfying $v_{k}=w_{k}^{\varepsilon}+y_{k}^{\varepsilon}+z_{k}^{\varepsilon}$,

$$
\left\{\begin{array}{l}
\left|\left\|y_{k}^{\varepsilon}\right\|_{1}-\mu\right| \leq \varepsilon,\left|\left\|z_{k}^{\varepsilon}\right\|_{1}-\left(M_{c}-\mu\right)\right| \leq \varepsilon,\left\|w_{k}^{\varepsilon}\right\|_{1} \leq \varepsilon \\
\lim _{k \rightarrow \infty} \operatorname{dist}\left(\operatorname{supp} y_{k}^{\varepsilon}, \operatorname{supp} z_{k}^{\varepsilon}\right)=\infty
\end{array}\right.
$$

for any $k \geq k_{0}$.

As usual we shall rule out the possible occurrence of vanishing and dichotomy. To this end we argue as in [27, Theorem II.1]. Let us first notice that by the scaling and non-negativity properties of the free energy, $(2.10)$ and $(3.6), \mathcal{F}\left[u\left(t_{k}\right)\right] \in\left[0, \mathcal{F}\left[u_{0}\right]\right]$ and

$$
\lim _{k \rightarrow \infty} \mathcal{F}\left[v_{k}\right]=\lim _{k \rightarrow \infty}\left\|u\left(t_{k}\right)\right\|_{m}^{-m} \mathcal{F}\left[u\left(t_{k}\right)\right]=0 .
$$

Consequently, since $\left\|v_{k}\right\|_{m}=1$ by the definition of $\lambda_{k}$, we have

$$
\lim _{k \rightarrow \infty} \mathcal{W}\left(v_{k}\right)=\lim _{k \rightarrow \infty} \frac{2}{c_{d}}\left(\frac{1}{m-1}\left\|v_{k}\right\|_{m}^{m}-\mathcal{F}\left[v_{k}\right]\right)=\frac{2}{c_{d}(m-1)}>0
$$

- Let us first show that vanishing does not take place and argue by contradiction. We split the non-local term $\mathcal{W}\left(v_{k}\right)$ in three parts. If $|x-y|$ is small, we control the corresponding term by the bound in $\mathrm{L}^{1} \cap \mathrm{L}^{m}$ of $v_{k}$. If $|x-y|$ is large the corresponding term is controlled by the $\mathrm{L}^{1}$-bound of $v_{k}$. And the remaining term converges to zero if we assume that vanishing occurs which contradicts (4.7). Indeed, if $q \in((d-1) /(d-2), d /(d-2))$ and $R>0$, it follows from the 
Hölder and Young inequalities that

$$
\begin{aligned}
& \mathcal{W}\left(v_{k}\right)= \iint_{\mathbb{R}^{d} \times \mathbb{R}^{d}} \frac{v_{k}(x) v_{k}(y)}{|x-y|^{d-2}} \mathbb{1}_{[0,1 / R]}(|x-y|) \mathrm{d} y \mathrm{~d} x+\iint_{\mathbb{R}^{d} \times \mathbb{R}^{d}} \frac{v_{k}(x) v_{k}(y)}{|x-y|^{d-2}} \mathbb{1}_{(1 / R, R)}(|x-y|) \mathrm{d} y \mathrm{~d} x \\
&+\iint_{\mathbb{R}^{d} \times \mathbb{R}^{d}} \frac{v_{k}(x) v_{k}(y)}{|x-y|^{d-2}} \mathbb{1}_{[R, \infty)}(|x-y|) \mathrm{d} y \mathrm{~d} x \\
& \leq\left\|v_{k}\right\|_{2 q /(2 q-1)}^{2}\left(\int_{\mathbb{R}^{d}}|x|^{-q(d-2)} \mathbb{1}_{[0,1 / R]}(|x|) \mathrm{d} x\right)^{1 / q}+R^{d-2} \int_{\mathbb{R}^{d}} v_{k}(x) \int_{B(x, R)} v_{k}(y) \mathrm{d} y \mathrm{~d} x \\
&+\frac{1}{R^{d-2}}\left(\int_{\mathbb{R}^{d}} v_{k}(x) \mathrm{d} x\right)^{2} \\
& \leq\left\|v_{k}\right\|_{m}^{m /[q(m-1)]}\left\|v_{k}\right\|_{1}^{[(2 q-1) / q]-[d /(q(d-2))]}\left(d \sigma_{d} \int_{0}^{1 / R} r^{d-1-q(d-2)} \mathrm{d} r\right)^{1 / q} \\
& \quad+R^{d-2} M_{c} \sup _{x \in \mathbb{R}^{d}} \int_{B(x, R)} v_{k}(y) \mathrm{d} y \mathrm{~d} x+\frac{M_{c}^{2}}{R^{d-2}} \\
& \leq C(q) \frac{1}{R^{[d-q(d-2)] / q}}+R^{d-2} M_{c} \sup _{x \in \mathbb{R}^{d}} \int_{B(x, R)} v_{k}(y) \mathrm{d} y \mathrm{~d} x+\frac{M_{c}^{2}}{R^{d-2}}
\end{aligned}
$$

We let $k \rightarrow \infty$ in the above inequality and use the vanishing assumption (4.4) to obtain that

$$
\limsup _{k \rightarrow \infty} \mathcal{W}\left(v_{k}\right) \leq C(q)\left(R^{2-d}+R^{-(d-q(d-2)) / q}\right) .
$$

We next let $R$ to infinity to conclude that $\mathcal{W}\left(v_{k}\right)$ converges to zero as $k \rightarrow \infty$ which contradicts (4.7).

- Let us next assume for contradiction that dichotomy takes place. We have

$$
\mathcal{W}\left(v_{k}\right)-\mathcal{W}\left(y_{k}^{\varepsilon}\right)-\mathcal{W}\left(z_{k}^{\varepsilon}\right)=-\mathcal{W}\left(w_{k}^{\varepsilon}\right)+\mathrm{I}_{1}+\mathrm{I}_{2}
$$

where

$$
\mathrm{I}_{1}:=2 \iint_{\mathbb{R}^{d} \times \mathbb{R}^{d}} y_{k}^{\varepsilon}(x) z_{k}^{\varepsilon}(y)|x-y|^{2-d} \mathrm{~d} x \mathrm{~d} y \text { and } \mathrm{I}_{2}:=2 \iint_{\mathbb{R}^{d} \times \mathbb{R}^{d}} v_{k}(x) w_{k}^{\varepsilon}(y)|x-y|^{2-d} \mathrm{~d} x \mathrm{~d} y .
$$

On the one hand, setting $d_{k}^{\varepsilon}:=\operatorname{dist}\left(\operatorname{supp} y_{k}^{\varepsilon}\right.$, supp $\left.z_{k}^{\varepsilon}\right)$, we have

$$
\begin{aligned}
\left|\mathrm{I}_{1}\right| \leq \int & \int_{\mathbb{R}^{d} \times \mathbb{R}^{d}} y_{k}^{\varepsilon}(x) z_{k}^{\varepsilon}(y) \mathbb{1}_{\left(0, d_{k}^{\varepsilon}\right)}(|x-y|)|x-y|^{2-d} \mathrm{~d} x \mathrm{~d} y \\
& +\iint_{\mathbb{R}^{d} \times \mathbb{R}^{d}} y_{k}^{\varepsilon}(x) z_{k}^{\varepsilon}(y) \mathbb{1}_{\left[d_{k}^{\varepsilon}, \infty\right)}(|x-y|)|x-y|^{2-d} \mathrm{~d} x \mathrm{~d} y .
\end{aligned}
$$

Thanks to the definition of $d_{k}^{\varepsilon}$ the first integral vanishes and we arrive at

$$
\left|\mathrm{I}_{1}\right| \leq M_{c}^{2}\left(d_{k}^{\varepsilon}\right)^{2-d} .
$$

On the other hand it follows from (4.2), (4.5), the HLS inequality (3.2) applied to $f=v_{k}, g=w_{k}^{\varepsilon}$, $\lambda=d-2$ and $p=q=2 d /(d+2)$, and the Hölder inequality with $1<2 d /(d+2)<m$ that

$$
\begin{aligned}
\left|\mathrm{I}_{2}\right| & \leq C_{\mathrm{HLS}}\left\|v_{k}\right\|_{2 d /(d+2)}\left\|w_{k}^{\varepsilon}\right\|_{2 d /(d+2)} \leq C_{\mathrm{HLS}}\left\|v_{k}\right\|_{m}^{m / 2}\left\|v_{k}\right\|_{1}^{1 / d}\left\|w_{k}^{\varepsilon}\right\|_{m}^{m / 2}\left\|w_{k}^{\varepsilon}\right\|_{1}^{1 / d} \\
& \leq C_{\mathrm{HLS}} M_{c}^{1 / d}\left\|w_{k}^{\varepsilon}\right\|_{m}^{m / 2} \varepsilon^{1 / d}
\end{aligned}
$$


and $0 \leq w_{k}^{\varepsilon} \leq v_{k}$ and (4.2) imply that $\left\|w_{k}^{\varepsilon}\right\|_{m} \leq 1$. Similarly by the variant of the HLS inequality (3.1), we obtain

$$
\left|\mathcal{W}\left(w_{k}^{\varepsilon}\right)\right| \leq C_{*}\left\|w_{k}^{\varepsilon}\right\|_{m}^{m} \varepsilon^{2 / d} \leq C_{*} \varepsilon^{2 / d} .
$$

Combining these estimates, we have thus shown that, given $\varepsilon \in(0,1)$, there exists $k_{\varepsilon} \geq \varepsilon^{-1}$ such that

$$
\left|\mathcal{W}\left(v_{k_{\varepsilon}}\right)-\mathcal{W}\left(y_{k_{\varepsilon}}^{\varepsilon}\right)-\mathcal{W}\left(z_{k_{\varepsilon}}^{\varepsilon}\right)\right| \leq \varepsilon^{1 / d} .
$$

Since $w_{k_{\varepsilon}}^{\varepsilon}$ is non-negative and the supports of $y_{k_{\varepsilon}}^{\varepsilon}$ and $z_{k_{\varepsilon}}^{\varepsilon}$ are disjoint we have

$$
\left\|y_{k_{\varepsilon}}^{\varepsilon}+z_{k_{\varepsilon}}^{\varepsilon}+w_{k_{\varepsilon}}^{\varepsilon}\right\|_{m}^{m} \geq\left\|y_{k_{\varepsilon}}^{\varepsilon}+z_{k_{\varepsilon}}^{\varepsilon}\right\|_{m}^{m}=\left\|y_{k_{\varepsilon}}^{\varepsilon}\right\|_{m}^{m}+\left\|z_{k_{\varepsilon}}^{\varepsilon}\right\|_{m}^{m},
$$

and we deduce from (4.8) that

$$
\begin{aligned}
\mathcal{F}\left[v_{k_{\varepsilon}}\right] & =\frac{1}{m-1}\left\|y_{k_{\varepsilon}}^{\varepsilon}+z_{k_{\varepsilon}}^{\varepsilon}+w_{k_{\varepsilon}}^{\varepsilon}\right\|_{m}^{m}-\frac{c_{d}}{2} \mathcal{W}\left(v_{k_{\varepsilon}}\right) \\
& \geq \frac{1}{m-1}\left(\left\|y_{k_{\varepsilon}}^{\varepsilon}\right\|_{m}^{m}+\left\|z_{k_{\varepsilon}}^{\varepsilon}\right\|_{m}^{m}\right)-\frac{c_{d}}{2} \mathcal{W}\left(y_{k_{\varepsilon}}^{\varepsilon}\right)-\frac{c_{d}}{2} \mathcal{W}\left(z_{k_{\varepsilon}}^{\varepsilon}\right)-\frac{c_{d}}{2} \varepsilon^{1 / d} \\
& \geq \mathcal{F}\left[y_{k_{\varepsilon}}^{\varepsilon}\right]+\mathcal{F}\left[z_{k_{\varepsilon}}^{\varepsilon}\right]-\frac{c_{d}}{2} \varepsilon^{1 / d} .
\end{aligned}
$$

The above inequality, (4.5), (4.6), and the non-negativity of $\mathcal{F}$ for functions with $\mathrm{L}^{1}$-norm lower or equal to $M_{c}$ then entail that

$$
\lim _{\varepsilon \rightarrow 0} \mathcal{F}\left[y_{k_{\varepsilon}}^{\varepsilon}\right]=\lim _{\varepsilon \rightarrow 0} \mathcal{F}\left[z_{k_{\varepsilon}}^{\varepsilon}\right]=0
$$

Now, (3.7) and (4.5) imply

$$
0=\lim _{\varepsilon \rightarrow 0} \mathcal{F}\left[y_{k_{\varepsilon}}^{\varepsilon}\right] \geq \lim _{\varepsilon \rightarrow 0} \frac{C_{*} c_{d}}{2}\left(M_{c}^{2 / d}-\left\|y_{k_{\varepsilon}}^{\varepsilon}\right\|_{1}^{2 / d}\right)\left\|y_{k_{\varepsilon}}^{\varepsilon}\right\|_{m}^{m} \geq \frac{C_{*} c_{d}}{2}\left(M_{c}^{2 / d}-\mu^{2 / d}\right) \lim _{\varepsilon \rightarrow 0}\left\|y_{k_{\varepsilon}}^{\varepsilon}\right\|_{m}^{m},
$$

and a similar inequality for $z_{k_{\varepsilon}}^{\varepsilon}$ (with $M_{c}-\mu$ instead of $\mu$ ), hence

$$
\lim _{\varepsilon \rightarrow 0}\left\|y_{k_{\varepsilon}}^{\varepsilon}\right\|_{m}^{m}=\lim _{\varepsilon \rightarrow 0}\left\|z_{k_{\varepsilon}}^{\varepsilon}\right\|_{m}^{m}=0
$$

Combining (4.9) and (4.10) gives

$$
\lim _{\varepsilon \rightarrow 0} \mathcal{W}\left(y_{k_{\varepsilon}}^{\varepsilon}\right)=\lim _{\varepsilon \rightarrow 0} \mathcal{W}\left(z_{k_{\varepsilon}}^{\varepsilon}\right)=0
$$

which, together with (4.8), implies that $\left(\mathcal{W}\left(v_{k_{\varepsilon}}\right)\right)_{\varepsilon}$ goes to 0 as $\varepsilon$ goes to infinity and contra$\operatorname{dicts}(4.7)$.

Having excluded the vanishing and dichotomy phenomena we thus conclude that there exists a sequence $\left(a_{k}\right)_{k}$ in $\mathbb{R}^{d}$ such that $\left(v_{k}\left(\cdot+a_{k}\right)\right)_{k}$ is tight, that is, satisfies (4.3).

Step 2 - Compactness in $\mathrm{L}^{m}$. We now aim at showing that a sub-sequence of $\left(v_{k}\left(\cdot+a_{k}\right)\right)_{k}$ converges in $\mathrm{L}^{1}\left(\mathbb{R}^{d}\right) \cap \mathrm{L}^{m}\left(\mathbb{R}^{d}\right)$ towards a minimiser of $\mathcal{F}$ in $\mathcal{Y}_{M_{c}}$. We set $V_{k}(x):=v_{k}\left(x+a_{k}\right)$ for $x \in \mathbb{R}^{d}$ and $k \geq 1$. By virtue of (4.2) we may assume (after possibly extracting a sub-sequence) that there is a non-negative $V_{\infty} \in \mathrm{L}^{m}\left(\mathbb{R}^{d}\right)$ such that

$$
V_{k} \rightarrow V_{\infty} \quad \text { weakly in } \mathrm{L}^{m}\left(\mathbb{R}^{d}\right)
$$

By (4.2), (4.3), and (4.11) we have $V_{\infty}$ is non-negative with $\left\|V_{\infty}\right\|_{1}=M_{c}$ and $\left\|V_{\infty}\right\|_{m} \leq 1$. 
To prove the convergence of $\mathcal{W}\left(V_{k}\right)$ to $\mathcal{W}\left(V_{\infty}\right)$, we proceed as in Step 1 and split $\mathbb{R}^{d} \times \mathbb{R}^{d}$ in three parts. If $q \in((d-1) /(d-2), d /(d-2))$ we have

$$
\begin{aligned}
\left|\mathcal{W}\left(V_{k}\right)-\mathcal{W}\left(V_{\infty}\right)\right| \leq \frac{2 M_{c}^{2}}{R^{d-2}}+C(q) R^{[q(2-d)+d] / q} & \\
& \quad\left|\iint_{\mathbb{R}^{d} \times \mathbb{R}^{d}}\left[V_{k}(x) V_{k}(y)-V_{\infty}(x) V_{\infty}(y)\right] \frac{\mathbb{1}_{(1 / R, R)}(|x-y|)}{|x-y|^{d-2}} \mathrm{~d} y \mathrm{~d} x\right| .
\end{aligned}
$$

Since $x \mapsto \mathbb{1}_{(1 / R, R)}(|x|)|x|^{2-d} \in \mathrm{L}^{1}\left(\mathbb{R}^{d}\right) \cap \mathrm{L}^{\infty}\left(\mathbb{R}^{d}\right)$, the weak convergence (4.11) ensures that $(x, y) \mapsto V_{k}(x) V_{k}(y)$ converges weakly toward $(x, y) \mapsto V_{\infty}(x) V_{\infty}(y)$ in $\mathrm{L}^{m}\left(\mathbb{R}^{d} \times \mathbb{R}^{d}\right)$ so that the last term of the right-hand side converges to zero as $k \rightarrow \infty$. Therefore

$$
\limsup _{k \rightarrow \infty}\left|\mathcal{W}\left(V_{k}\right)-\mathcal{W}\left(V_{\infty}\right)\right| \leq C(q)\left(R^{2-d}+R^{-[d-q(d-2)] / q}\right)
$$

We then let $R \rightarrow \infty$ to obtain

$$
\lim _{k \rightarrow \infty} \mathcal{W}\left(V_{k}\right)=\mathcal{W}\left(V_{\infty}\right)
$$

Owing to the lower semi-continuity of the $\mathrm{L}^{m}$-norm and (4.6) we have

$$
\mathcal{F}\left[V_{\infty}\right] \leq \frac{1}{m-1} \liminf _{k \rightarrow \infty}\left\|V_{k}\right\|_{m}^{m}-\frac{c_{d}}{2} \lim _{k \rightarrow \infty} \mathcal{W}\left(V_{k}\right) \leq \lim _{k \rightarrow \infty} \mathcal{F}\left[V_{k}\right]=0
$$

while Proposition 3.4 warrants that $\mathcal{F}\left[V_{\infty}\right] \geq 0$ as $V_{\infty} \in \mathcal{Y}_{M_{c}}$. Consequently, $\mathcal{F}\left[V_{\infty}\right]=0$ and the strong convergence of $\left(V_{k}\right)_{k}$ to $V_{\infty}$ in $\mathrm{L}^{m}\left(\mathbb{R}^{d}\right)$ readily follows: indeed,

$$
\frac{1}{m-1}\left\|V_{\infty}\right\|_{m}^{m}=\mathcal{F}\left[V_{\infty}\right]+\frac{c_{d}}{2} \mathcal{W}\left(V_{\infty}\right)=\lim _{k \rightarrow \infty}\left(\mathcal{F}\left[V_{k}\right]+\frac{c_{d}}{2} \mathcal{W}\left(V_{k}\right)\right)=\frac{1}{m-1} \lim _{k \rightarrow \infty}\left\|V_{k}\right\|_{m}^{m}
$$

We have thus shown that $V_{\infty}$ is a minimiser of $\mathcal{F}$ in $\mathcal{Y}_{M_{c}}$ with the additional property $\left\|V_{\infty}\right\|_{m}=$ 1. Furthermore, according to the characterisation of the minimisers given in Proposition 3.5, there exists $y_{0} \in \mathbb{R}^{d}$ such that $V_{\infty}\left(\cdot+y_{0}\right)=: V$ is the unique radially symmetric minimiser of $\mathcal{F}$ in $\mathcal{Y}_{M_{c}}$ with $\|V\|_{m}=1$. Coming back to the original variables we have proved that $\left(x \mapsto \lambda_{k}^{d} u\left(t_{k}, \lambda_{k}\left(x+a_{k}+y_{0}\right)\right)\right)_{k}$ converges to $V$ in $\mathrm{L}^{1}\left(\mathbb{R}^{d}\right)$ and $\mathrm{L}^{m}\left(\mathbb{R}^{d}\right)$. Setting $x_{k}=\lambda_{k}\left(a_{k}+y_{0}\right)$ gives

$$
\lim _{k \rightarrow \infty} \int_{\mathbb{R}^{d}}\left|u\left(t_{k}, x+x_{k}\right)-\frac{1}{\lambda_{k}^{d}} V\left(\frac{x}{\lambda_{k}}\right)\right| \mathrm{d} x=0,
$$

and thus the first assertion of Proposition 4.5.

Step 3 - Convergence of $\left(x_{k}\right)_{k}$. We first note that

$$
\int_{\mathbb{R}^{d}} x u(t, x) \mathrm{d} x=\int_{\mathbb{R}^{d}} x u_{0}(x) \mathrm{d} x
$$

for $t \in\left[0, T_{\omega}\right)$ so that we have also

$$
\bar{x}=\frac{1}{M_{c}} \int_{\mathbb{R}^{d}} x u(t, x) \mathrm{d} x
$$


for $t \in\left[0, T_{\omega}\right)$ by $(1.7)$. Next, for $\varepsilon \in(0,1)$, we have

$$
\begin{aligned}
\left|\left(\bar{x}-x_{k}\right) \int_{B\left(x_{k}, \varepsilon\right)} u\left(t_{k}, x\right) \mathrm{d} x\right| & \leq\left|\int_{B\left(x_{k}, \varepsilon\right)}(\bar{x}-x) u\left(t_{k}, x\right) \mathrm{d} x\right|+\left|\int_{B\left(x_{k}, \varepsilon\right)}\left(x-x_{k}\right) u\left(t_{k}, x\right) \mathrm{d} x\right| \\
& \leq \int_{\left\{\left|x-x_{k}\right| \geq \varepsilon\right\}}|\bar{x}-x| u\left(t_{k}, x\right) \mathrm{d} x+\varepsilon M_{c} \\
& \leq \int_{\left\{\left|x-x_{k}\right| \geq \varepsilon,|x-\bar{x}| \leq 1 / \varepsilon\right\}}|\bar{x}-x| u\left(t_{k}, x\right) \mathrm{d} x \\
& +\int_{\left\{\left|x-x_{k}\right| \geq \varepsilon,|x-\bar{x}|>1 / \varepsilon\right\}}|\bar{x}-x| u\left(t_{k}, x\right) \mathrm{d} x+\varepsilon M_{c} \\
& \leq \frac{1}{\varepsilon} \int_{\left\{\left|x-x_{k}\right| \geq \varepsilon\right\}} u\left(t_{k}, x\right) \mathrm{d} x+\varepsilon\left(\mathcal{M}_{2}+M_{c}\right) \\
& \leq \frac{1}{\varepsilon} \int_{\mathbb{R}^{d}}\left|u\left(t_{k}, y+x_{k}\right)-\frac{1}{\lambda_{k}^{d}} V\left(\frac{y}{\lambda_{k}}\right)\right| \mathrm{d} y \\
& +\frac{1}{\varepsilon} \int_{\left\{|x| \geq \varepsilon / \lambda_{k}\right\}} V(y) \mathrm{d} y+\varepsilon\left(\mathcal{M}_{2}+M_{c}\right) .
\end{aligned}
$$

Since $\lambda_{k} \rightarrow 0$ as $k \rightarrow \infty$ we infer from (4.12) and the integrability of $V$ that

$$
\limsup _{k \rightarrow \infty}\left|\left(\bar{x}-x_{k}\right) \int_{B\left(x_{k}, \varepsilon\right)} u\left(t_{k}, x\right) \mathrm{d} x\right| \leq \varepsilon\left(\mathcal{M}_{2}+M_{c}\right) .
$$

Using once more (4.12) we readily deduce that

$$
\lim _{k \rightarrow \infty} \int_{B\left(x_{k}, \varepsilon\right)} u\left(t_{k}, x\right) \mathrm{d} x=\int_{\mathbb{R}^{d}} V(x) \mathrm{d} x=M_{c} .
$$

Combining the previous two limits gives

$$
M_{c} \limsup _{k \rightarrow \infty}\left|\bar{x}-x_{k}\right| \leq \varepsilon\left(\mathcal{M}_{2}+M_{c}\right),
$$

whence the last assertion of Proposition 4.5 by letting $\varepsilon \rightarrow 0$.

For radially symmetric solutions we can remove the additional assumption on the second moment.

Corollary 4.6 (Radially symmetric blow-up) Let $u_{0}$ be a radially symmetric initial condition satisfying (1.2) with $\left\|u_{0}\right\|_{1}=M_{c}$ and consider a radially symmetric free energy solution $u$ to (1.3) on $\left[0, T_{\omega}\right)$ with initial condition $u_{0}$ and $T_{\omega} \in(0, \infty]$ and such that $\|u(t)\|_{m} \rightarrow \infty$ as $t \nearrow T_{\omega}$. If $\left(t_{k}\right)_{k}$ is a sequence of positive real numbers such that $t_{k} \rightarrow T_{\omega}$ as $k \rightarrow \infty$, there is a sub-sequence $\left(t_{k_{j}}\right)_{j}$ of $\left(t_{k}\right)_{k}$ such that

$$
\lim _{j \rightarrow \infty} \int_{\mathbb{R}^{d}}\left|u\left(t_{k_{j}}, x\right)-\frac{1}{\lambda_{k_{j}}^{d}} V\left(\frac{x}{\lambda_{k_{j}}}\right)\right| \mathrm{d} x=0,
$$

where $\lambda_{k}:=\left\|u\left(t_{k}\right)\right\|_{m}^{-m /(d-2)}$ and $V$ is the unique radially symmetric minimiser of $\mathcal{F}$ in $\mathcal{Y}_{M_{c}}$ such that $\|V\|_{m}=1$.

Proof. The only modification of the proof of Proposition 4.5 is to show that we can choose $a_{k}=0$ for all $k$ at the end of Step 1. Indeed, we claim that if $\varepsilon \in\left(0, M_{c} / 4\right)$ we have $\left|a_{k}\right| \leq R_{\varepsilon}$, where 
$a_{k}$ and $R_{\varepsilon}$ are defined in (4.3). Otherwise $B\left(a_{k}, R_{\varepsilon}\right)$ and $B\left(-a_{k}, R_{\varepsilon}\right)$ are disjoint and the radial symmetry of $v_{k}$ and (4.3) imply that

$$
M_{c} \geq \int_{B\left(a_{k}, R_{\varepsilon}\right) \cup B\left(-a_{k}, R_{\varepsilon}\right)} v_{k}(x) \mathrm{d} x=2 \int_{B\left(a_{k}, R_{\varepsilon}\right)} v_{k}(x) \mathrm{d} x \geq 2\left(M_{c}-\varepsilon\right) \geq \frac{3 M_{c}}{2}
$$

and a contradiction. Therefore $B\left(a_{k}, R_{\varepsilon}\right) \subset B\left(0,2 R_{\varepsilon}\right)$ and thus

$$
\int_{B\left(0,2 R_{\varepsilon}\right)} v_{k}(x) \mathrm{d} x \geq M_{c}-\varepsilon
$$

by (4.3).

\subsubsection{When would it blow-up?}

Proposition 4.7 (Global existence in the critical case) Let $u_{0}$ be an initial condition satisfying (1.2) with $\left\|u_{0}\right\|_{1}=M_{c}$ and consider a free energy solution $u$ to (1.3) on $\left[0, T_{\omega}\right)$ with initial condition $u_{0}$ and $T_{\omega} \in(0, \infty]$. Then $T_{\omega}=\infty$.

The proof of this proposition relies on Proposition 4.5 and the following control of the behaviour of free energy solutions for large $x$ :

Lemma 4.8 (Control of the tail) Consider a free energy solution $u$ to (1.3) on $\left[0, T_{\omega}\right)$ with initial condition $u_{0}$ satisfying $(1.2)$ and $T_{\omega} \in(0, \infty]$. If $t \longmapsto \mathcal{F}[u(t)]$ is bounded from below in $[0, T)$ for some $T \leq T_{\omega}$ then

$$
\lim _{R \rightarrow \infty} \sup _{t \in[0, T)} \int_{\{|x|>R\}}|x|^{2} u(t, x) \mathrm{d} x=0 .
$$

Proof. Consider a non-decreasing function $\xi \in \mathcal{C}^{\infty}(\mathbb{R})$ such that $\xi(r)=0$ for $|r| \leq 1$ and $\xi(r)=1$ for $|r| \geq 2$ and define

$$
\Phi_{R}(r)=r \xi^{4}\left(\frac{r}{R}\right) \quad \text { for } \quad r \in \mathbb{R} \text { and } \quad R>0 .
$$

The support of $\Phi_{R}$ is included in $\mathbb{R}^{d} \backslash B(0, R)$ and, introducing

$$
\mathcal{I}_{R}(t):=\int_{\{|x|>R\}}\left|\left(\frac{2 m}{2 m-1} \nabla u^{(2 m-1) / 2}-u^{1 / 2} \nabla \phi\right)(t, x)\right|^{2} \mathrm{~d} x,
$$

we have

$$
\begin{aligned}
\frac{\mathrm{d}}{\mathrm{d} t} \int_{\mathbb{R}^{d}} & \Phi_{R}\left(|x|^{2}\right) u(t, x) \mathrm{d} x \\
& =-\int_{\mathbb{R}^{d}} 2 x \Phi_{R}^{\prime}\left(|x|^{2}\right)\left(\frac{2 m}{2 m-1} \nabla u^{(2 m-1) / 2}-u^{1 / 2} \nabla \phi\right)(t, x) \mathrm{d} x \\
& \leq 2\left(\int_{\mathbb{R}^{d}}|x|^{2}\left|\Phi_{R}^{\prime}\left(|x|^{2}\right)\right|^{2} u(t, x) \mathrm{d} x\right)^{1 / 2} \mathcal{I}_{R}(t)^{1 / 2}
\end{aligned}
$$

By the definition of $\Phi_{R}$, we have

$$
\begin{aligned}
\left|\Phi_{R}^{\prime}(r)\right|^{2} & \leq\left|\xi^{4}\left(\frac{r}{R}\right)+4 \frac{r}{R} \xi^{3}\left(\frac{r}{R}\right) \xi^{\prime}\left(\frac{r}{R}\right)\right|^{2} \leq 2 \xi^{8}\left(\frac{r}{R}\right)+32\left(\frac{r}{R}\right)^{2} \xi^{6}\left(\frac{r}{R}\right)\left|\xi^{\prime}\left(\frac{r}{R}\right)\right|^{2} \\
& \leq \xi^{4}\left(\frac{r}{R}\right)\left(2+32 \sup _{z \in \mathbb{R}}\left|z \xi^{\prime}(z)\right|^{2}\right)
\end{aligned}
$$


so that $r\left|\Phi_{R}^{\prime}(r)\right|^{2} \leq C \Phi_{R}(r)$ for $r \in \mathbb{R}$. Therefore, for any $t \in[0, T)$,

$$
\frac{\mathrm{d}}{\mathrm{d} t} \int_{\mathbb{R}^{d}} \Phi_{R}\left(|x|^{2}\right) u(t, x) \mathrm{d} x \leq C\left(\int_{\mathbb{R}^{d}} \Phi_{R}\left(|x|^{2}\right) u(t, x) \mathrm{d} x\right)^{1 / 2} \mathcal{I}_{R}(t)^{1 / 2}
$$

hence

$$
\left(\int_{\mathbb{R}^{d}} \Phi_{R}\left(|x|^{2}\right) u(t, x) \mathrm{d} x\right)^{1 / 2} \leq\left(\int_{\mathbb{R}^{d}} \Phi_{R}\left(|x|^{2}\right) u_{0}(x) \mathrm{d} x\right)^{1 / 2}+\frac{C}{2} \int_{0}^{t} \mathcal{I}_{R}^{1 / 2}(s) \mathrm{d} s .
$$

Now, since $\mathcal{F}[u(t)]$ is bounded from below in $[0, T)$, we have

$$
\int_{0}^{T} \int_{\mathbb{R}^{d}}\left|\left(\frac{2 m}{2 m-1} \nabla u^{(2 m-1) / 2}-u^{1 / 2} \nabla \phi\right)(s, x)\right|^{2} \mathrm{~d} x \mathrm{~d} s \leq \sup _{t \in[0, T)}\left\{\mathcal{F}\left[u_{0}\right]-\mathcal{F}[u(t)]\right\}<\infty,
$$

so that

$$
\lim _{R \rightarrow \infty} \int_{0}^{T} \mathcal{I}_{R}^{1 / 2}(s) \mathrm{d} s=0
$$

by the Lebesgue dominated convergence theorem. Therefore,

$$
\limsup _{R \rightarrow \infty} \sup _{t \in[0, T)} \int_{\mathbb{R}^{d}} \Phi_{R}\left(|x|^{2}\right) u(t, x) \mathrm{d} x=0
$$

from which the lemma follows.

Proof of Proposition 4.7. Assume for contradiction that $T_{\omega}$ is finite and let $\left(t_{k}\right)_{k}$ be a sequence of positive real numbers such that $t_{k} \rightarrow T_{\omega}$ as $k \rightarrow \infty$. Observe that Theorem 2.4 entails that $\|u(t)\|_{m} \rightarrow \infty$ as $t \rightarrow T_{\omega}$. On the one hand we infer from the nature of the blow-up given in Proposition 4.5 that there are a sub-sequence of $\left(t_{k}\right)_{k}$ (not relabelled) and a sequence $\left(x_{k}\right)_{k}$ in $\mathbb{R}^{d}$ such that

$$
\begin{aligned}
& \lim _{k \rightarrow \infty} x_{k}=\bar{x}:=\frac{1}{M_{c}} \int_{\mathbb{R}^{d}} x u_{0}(x) \mathrm{d} x \\
& \lim _{k \rightarrow \infty} \int_{\mathbb{R}^{d}}\left|u\left(t_{k}, x+x_{k}\right)-\frac{1}{\lambda_{k}^{d}} V\left(\frac{x}{\lambda_{k}}\right)\right| \mathrm{d} x=0
\end{aligned}
$$

with $\lambda_{k}:=\left\|u\left(t_{k}\right)\right\|_{m}^{-m /(d-2)}$. On the other hand it follows from Proposition 3.4 and Lemma 4.1 that $\mathcal{F}[u(t)] \geq 0$ for $t \in\left[0, T_{\omega}\right)$ so that

$$
\begin{aligned}
\int_{\mathbb{R}^{d}}|x-\bar{x}|^{2} u(t, x) \mathrm{d} x & =\int_{\mathbb{R}^{d}}|x-\bar{x}|^{2} u_{0}(x) \mathrm{d} x+2(d-2) \int_{0}^{t} \mathcal{F}[u(s)] \mathrm{d} s \\
& \geq \int_{\mathbb{R}^{d}}|x-\bar{x}|^{2} u_{0}(x) \mathrm{d} x>0
\end{aligned}
$$

and Lemma 4.8 may be applied to obtain

$$
\lim _{R \rightarrow \infty} \sup _{t \in\left[0, T_{\omega}\right)} \int_{\{|x|>R\}}|x|^{2} u(t, x) \mathrm{d} x=0 .
$$


Now, for $k \geq 1$ and $R \geq|\bar{x}|$ we have

$$
\begin{aligned}
\int_{\mathbb{R}^{d}}|x-\bar{x}|^{2} u\left(t_{k}, x\right) \mathrm{d} x & \leq 2 \int_{\{|x-\bar{x}| \geq 2 R\}}\left(|x|^{2}+|\bar{x}|^{2}\right) u\left(t_{k}, x\right) \mathrm{d} x \\
& +\int_{\{|x-\bar{x}|<2 R\}}|x-\bar{x}|^{2}\left[u(t, x)-\frac{1}{\lambda_{k}^{d}} V\left(\frac{x-x_{k}}{\lambda_{k}}\right)\right] \mathrm{d} x \\
& +\int_{\{|x-\bar{x}|<2 R\}} \frac{|x-\bar{x}|^{2}}{\lambda_{k}^{d}} V\left(\frac{x-x_{k}}{\lambda_{k}}\right) \mathrm{d} x \\
& \leq 4 \int_{\{|x| \geq R\}}|x|^{2} u\left(t_{k}, x\right) \mathrm{d} x+R^{2} \int_{\mathbb{R}^{d}}\left|u(t, x)-\frac{1}{\lambda_{k}^{d}} V\left(\frac{x-x_{k}}{\lambda_{k}}\right)\right| \mathrm{d} x \\
& +\int_{\mathbb{R}^{d}}\left|\lambda_{k} x+x_{k}-\bar{x}\right|^{2} V(x) \mathrm{d} x \\
& \leq 4 \sup _{t \in\left[0, T_{\omega}\right)} \int_{\{|x| \geq R\}}|x|^{2} u(t, x) \mathrm{d} x+R^{2} \int_{\mathbb{R}^{d}}\left|u(t, x)-\frac{1}{\lambda_{k}^{d}} V\left(\frac{x-x_{k}}{\lambda_{k}}\right)\right| \mathrm{d} x \\
& +2\left|x_{k}-\bar{x}\right|^{2} M_{c}+2 \lambda_{k}^{2} \int_{\mathbb{R}^{d}}|x|^{2} V(x) \mathrm{d} x .
\end{aligned}
$$

Owing to (4.13), (4.14), and the convergence of $\left(\lambda_{k}\right)_{k}$ to zero we may let $k \rightarrow \infty$ in the previous inequality to obtain

$$
\limsup _{k \rightarrow \infty} \int_{\mathbb{R}^{d}}|x-\bar{x}|^{2} u\left(t_{k}, x\right) \mathrm{d} x \leq 4 \sup _{t \in\left[0, T_{\omega}\right)} \int_{\{|x| \geq R\}}|x|^{2} u(t, x) \mathrm{d} x .
$$

We next pass to the limit as $R \rightarrow \infty$ with the help of (4.16) to conclude that

$$
\lim _{k \rightarrow \infty} \int_{\mathbb{R}^{d}}|x-\bar{x}|^{2} u\left(t_{k}, x\right) \mathrm{d} x=0,
$$

which contradicts (4.15).

\subsubsection{Does it blow-up?}

Let us first note that Proposition 4.5 allows us to describe the nature of the blow-up when it occurs. We define the two following statements:

$$
\begin{aligned}
& \text { There exists }\left(t_{k}\right)_{k} \nearrow \infty \text { such that }\left(\left\|u\left(t_{k}\right)\right\|_{m}\right)_{k} \text { is bounded } \\
& \qquad \mathcal{M}_{2}^{\infty}:=\lim _{t \rightarrow \infty} \int_{\mathbb{R}^{d}}|x|^{2} u(t, x) \mathrm{d} x<\infty
\end{aligned}
$$

- If [not (S1)] and (S2): By Proposition 4.5, the solution blows up as a Dirac mass at the centre of mass as $t$ goes to infinity. Moreover, the blow-up profile is described by the minimisers of $\mathcal{F}$ for the critical mass.

- If (S1) and (S2): By the virial identity Lemma 4.1, $\mathcal{F}\left[u\left(t_{k}\right)\right] \rightarrow 0$ so that $\left(u\left(t_{k}\right)\right)_{k}$ is a minimising sequence for $\mathcal{F}$ in $\mathcal{Y}_{M_{c}}$. We expect that it converges to the minimiser of $\mathcal{F}$ in $\mathcal{Y}_{M_{c}}$ with centre of mass $\bar{x}$ defined in (4.1) and second moment $\mathcal{M}_{2}^{\infty}$.

- If [not (S1)] and [not (S2)]: By Proposition 4.5, the solution blows up as a Dirac mass. The blow-up profile is described by the minimisers of $\mathcal{F}$ for the critical mass. However, we cannot prevent the escape at infinity of the Dirac mass. 
- If (S1) and [not (S2)]: No precise information can be deduced in this case. We cannot even rule out the possibility of the existence of another sequence of times for which the $\mathrm{L}^{m}$-norm diverges.

In the radially symmetric case, if the initial condition is less concentrated than one of the stationary solutions, then we strongly believe that such a property remains true for all times, thus excluding the formation of a Dirac mass. According to the above discussion this prevents the blow-up of the $\mathrm{L}^{m}$-norm in infinite time and give an example where (S1) and (S2) hold true. This is in sharp contrast with the two-dimensional PKS case where infinite time blow-up always occurs, see $[4,6]$.

\section{$5 \quad$ Sub-critical self-similar solutions}

For the classical PKS system in space dimension $d=2$ (and $m=1$ ), the large time behaviour of solutions with subcritical mass is described by self-similar solutions [4, 7]. This fact and the already mentioned scale invariance of (1.3) motivate us to look for self-similar solutions to (1.3) with subcritical mass. To this end, we first recall the by-now classical self-similar scaling leading to a non-linear Fokker-Planck equation as in [13] for the porous medium equation: Let us define $\rho$ by $\rho(s, y):=\mathrm{e}^{d s} u\left(\beta(s), \mathrm{e}^{s} y\right)$ and $c(s, y)=(\mathcal{K} * \rho)(s, y)$ with $\beta$ strictly increasing to be chosen. Then, it is straightforward to check that

$$
\left\{\begin{aligned}
\frac{\partial \rho}{\partial s}(s, y) & =\operatorname{div}\left[y \rho(s, y)+\beta^{\prime}(t)\left\{\mathrm{e}^{-\lambda t} \nabla \rho^{m}(s, y)-\mathrm{e}^{-d t} \rho(s, y) \nabla c(s, y)\right\}\right], & & s>0, y \in \mathbb{R}^{d}, \\
-\Delta c(s, y) & =\rho(s, y), & & s>0, y \in \mathbb{R}^{d}, \\
\rho(0, y) & =u_{0}(y) \geq 0, & & y \in \mathbb{R}^{d},
\end{aligned}\right.
$$

with $\lambda=d(m-1)+2=d$. Let us remark that our choice of the exponent $m$ is the only one for which the scaling of the aggregation and the diffusion terms matches as mentioned in the introduction. In this case, $\beta^{\prime}(s)=\mathrm{e}^{d s}$ determines the change of variables and the final scaled equation reads:

$$
\left\{\begin{aligned}
\frac{\partial \rho}{\partial s}(s, y) & =\operatorname{div}\left[y \rho(s, y)+\nabla \rho^{m}(s, y)-\rho(s, y) \nabla c(s, y)\right], & & s>0, y \in \mathbb{R}^{d} \\
-\Delta c(s, y) & =\rho(s, y), & & s>0, y \in \mathbb{R}^{d} \\
\rho(0, y) & =u_{0}(y) \geq 0, & & y \in \mathbb{R}^{d} .
\end{aligned}\right.
$$

Self-similar solutions to (1.3) now correspond to stationary solutions to (5.1) and we will establish the existence of the latter by a variational technique. More precisely, we will show the existence of minimisers for the free energy $\mathcal{G}$ associated to the rescaled problem (5.1) given by

$$
\mathcal{G}[h]:=\mathcal{F}[h]+\frac{1}{2} M_{2}[h] \quad \text { with } \quad M_{2}[h]:=\int_{\mathbb{R}^{d}}|x|^{2}|h(x)| \mathrm{d} x
$$

for $h \in \mathrm{L}^{1}\left(\mathbb{R}^{d} ;\left(1+x^{2}\right) \mathrm{d} x\right) \cap \mathrm{L}^{m}\left(\mathbb{R}^{d}\right)$. For $M>0$, we define

$$
\nu_{M}:=\inf \left\{\mathcal{G}[h]: h \in \mathcal{Z}_{M}\right\} \quad \text { with } \quad \mathcal{Z}_{M}:=\left\{h \in \mathcal{Y}_{M}: M_{2}[h]<\infty\right\}
$$

We first establish the following analogue of Proposition 3.4. 
Proposition 5.1 (Infimum of the rescaled free energy) For $M>0$ and $h \in \mathcal{Z}_{M}$ we have

$$
\mathcal{G}[h] \geq \frac{C_{*} c_{d}}{2}\left(M_{c}^{2 / d}-M^{2 / d}\right)\|h\|_{m}^{m}+\frac{1}{2} M_{2}[h] .
$$

In addition,

$$
\begin{cases}\nu_{M}>0 & \text { if } M<M_{c}, \\ \nu_{M_{c}}=0, & \\ \nu_{M}=-\infty & \text { if } M>M_{c} .\end{cases}
$$

Proof. The inequality (5.2) readily follows from (3.7) and the definition of $\mathcal{G}$. Consider next $M \geq M_{c}$ and put

$$
h_{R}(x):= \begin{cases}\frac{M}{M_{c}} \frac{1}{R^{d}} \zeta^{d /(d-2)}\left(\frac{x}{R^{d}}\right) & \text { if } x \in B(0, R), \\ 0 & \text { if } x \in \mathbb{R}^{d} \backslash B(0, R),\end{cases}
$$

where the function $\zeta$ is defined in Proposition 3.5 and $R>0$. We compute $\mathcal{G}\left[h_{R}\right]$ and use the property $\mathcal{F}\left[\zeta^{d /(d-2)}\right]=0$ to obtain

$$
\nu_{M} \leq \mathcal{G}\left[h_{R}\right]=\left(\frac{M}{M_{c}}\right)^{2} R^{2-d}\left[\frac{M_{c}}{2 M} R^{d} M_{2}\left[\zeta^{d /(d-2)}\right]-\left(1-\left(\frac{M_{c}}{M}\right)^{2-m}\right) \frac{\left\|\zeta^{d /(d-2)}\right\|_{m}^{m}}{m-1}\right]
$$

Now, either $M>M_{c}$ and the right-hand side of the above inequality diverges to $-\infty$ as $R \rightarrow 0$ since $d>2$ and $m<2$. Consequently $\nu_{m}=-\infty$ in that case. Or $M=M_{c}$ and we may let $R \rightarrow 0$ in the above inequality to obtain that $\nu_{M_{c}} \leq 0$. Since $\mathcal{G}$ is non-negative by Proposition 3.4, we conclude that $\nu_{M_{c}}=0$.

Finally, assume for contradiction that $\nu_{M}=0$ for some $M<M_{c}$ and let $\left(h_{k}\right)_{k}$ be a minimising sequence for $\mathcal{G}$ in $\mathcal{Z}_{M}$. Since $\mathcal{G}\left[h_{k}\right] \geq \mathcal{G}\left[\left|h_{k}\right|\right],\left(\left|h_{k}\right|\right)_{k}$ is also a minimising sequence for $\mathcal{G}$ in $\mathcal{Z}_{M}$ and we infer from (5.2) that

$$
\lim _{k \rightarrow \infty}\left(\left\|h_{k}\right\|_{m}+\frac{1}{2} M_{2}\left[h_{k}\right]\right)=0
$$

By Vitali's theorem $\left(\left|h_{k}\right|\right)_{k}$ converges towards zero in $\mathrm{L}^{1}\left(\mathbb{R}^{d}\right)$ which contradicts the fact that $\left\|h_{k}\right\|_{\mathrm{L}^{1}}=M$ for all $k \geq 1$. Therefore $\nu_{M} \neq 0$ and the non-negativity of $\mathcal{G}$ in $\mathcal{Z}_{M}$ entails that $\nu_{M}>0$.

We next identify the minimisers of $\mathcal{G}$ in $\mathcal{Z}_{M}$ for $M \in\left(0, M_{c}\right)$.

Theorem 5.2 (Identification of minimisers) If $M \in\left(0, M_{c}\right)$ there is a unique minimiser $W_{M}$ of $\mathcal{G}$ in $\mathcal{Z}_{M}$. In addition, $W_{M}$ is non-negative radially symmetric and non-increasing and there is a unique $\varrho_{M}>0$ such that $W_{M}(x)=0$ for $|x| \geq \varrho_{M}$ and $\xi_{M}:=W_{M}^{m-1}$ solves

$$
\Delta \xi_{M}+\frac{m-1}{m}\left(\xi_{M}^{1 /(m-1)}+d\right)=0 \quad \text { in } \quad B\left(0, \varrho_{M}\right) \quad \text { with } \quad \xi_{M}=0 \quad \text { on } \quad \partial B\left(0, \varrho_{M}\right) .
$$

Several steps are required to perform the proof of Theorem 5.2 which borrows several arguments from $[24,26]$. We first establish the existence of minimisers of $\mathcal{G}$ in $\mathcal{Z}_{M}$ for $M \in\left(0, M_{c}\right)$.

Lemma 5.3 (Existence of minimisers) Consider $M \in\left(0, M_{c}\right)$. The functional $\mathcal{G}$ has at least a minimiser in $\mathcal{Z}_{M}$. In addition, every minimiser of $\mathcal{G}$ in $\mathcal{Z}_{M}$ is non-negative radially symmetric and non-increasing. 
Proof. We first recall that, if $h \in \mathrm{L}^{1}\left(\mathbb{R}^{d} ;\left(1+|x|^{2}\right) \mathrm{d} x\right)$ and $h^{*}$ denotes its symmetric decreasing rearrangement, then $M_{2}\left[h^{*}\right] \leq M_{2}[h]$. Thanks to this property, we may next argue as in the proof of Lemma 3.3 to conclude that there is at least a minimiser of $\mathcal{G}$ in $\mathcal{Z}_{M}$.

Next, let $W$ be a minimiser of $\mathcal{G}$ in $\mathcal{Z}_{M}$ and denote by $W^{*}$ its symmetric decreasing rearrangement. As

$$
\left\|W^{*}\right\|_{1}=\|W\|_{1}, \quad\left\|W^{*}\right\|_{m}=\|W\|_{m}, \quad \text { and } \quad M_{2}\left[W^{*}\right] \leq M_{2}[W],
$$

$W^{*}$ belongs to $\mathcal{Z}_{M}$. In addition, by Riesz's rearrangement inequality [24, Lemma 2.1$], \mathcal{W}(W) \leq$ $\mathcal{W}\left(W^{*}\right)$. Consequently, $\nu_{M}=\mathcal{G}[W] \geq \mathcal{G}\left[W^{*}\right]$ and $W^{*}$ is also a minimiser of $\mathcal{G}$ in $\mathcal{Z}_{M}$. This last property entails that

$$
M_{2}\left[W^{*}\right]=M_{2}[W] \text { and } \mathcal{W}\left(W^{*}\right)=\mathcal{W}(W) .
$$

Using once more [24, Lemma 2.1 (ii)] we deduce from $\mathcal{W}\left(W^{*}\right)=\mathcal{W}(W)$ that there is $y \in \mathbb{R}^{d}$ such that $W(x)=W^{*}(x+y)$ for $x \in \mathbb{R}^{d}$. Then $M_{2}\left[W^{*}\right]=M_{2}[W]$ implies that $y=0$, which completes the proof.

We are thus left with the uniqueness issue to complete the proof of Theorem 5.2. To this end we adapt the proof in [26, Section IV.B] and first proceed as in the proof of Proposition 3.5 to identify the Euler-Lagrange equation satisfied by the minimisers of $\mathcal{G}$ in $\mathcal{Z}_{M}$.

Lemma 5.4 Consider $M \in\left(0, M_{c}\right)$ and let $W$ be a minimiser of $\mathcal{G}$ in $\mathcal{Z}_{M}$. Then there is $\varrho>0$ such that $W(x)=0$ if $|x| \geq \varrho$ and $\xi:=W^{m-1}$ is a non-negative radially symmetric and non-increasing classical solution to

$$
\Delta \xi+\frac{m-1}{m}\left(\xi^{1 /(m-1)}+d\right)=0 \quad \text { in } \quad B(0, \varrho) \quad \text { with } \quad \xi=0 \quad \text { on } \quad \partial B(0, \varrho) .
$$

In addition,

$$
\frac{m}{m-1} W^{m-1}=\left(\mathcal{K} * W-\frac{|x|^{2}}{2}+\frac{1}{2}+\frac{m}{m-1} M^{m-1}-\frac{c_{d}}{M} \mathcal{W}(W)\right)_{+} \quad \text { a.e. in } \mathbb{R}^{d}
$$

Additional properties of minimisers of $\mathcal{G}$ in $\mathcal{Z}_{M}$ can be deduced from Lemma 5.4.

Lemma 5.5 Consider $M \in\left(0, M_{c}\right)$ and let $W$ be a minimiser of $\mathcal{G}$ in $\mathcal{Z}_{M}$. Then

$$
\begin{aligned}
M_{2}[W]=(d-2) \mathcal{F}[W] & =2(m-1) \nu_{M}, \\
\frac{2 m}{m-1}\|W\|_{m}^{m}+M_{2}[W] & =\frac{2 m}{m-1} M^{m}+M .
\end{aligned}
$$

Proof. We proceed as in [26, Lemma 6]. By Lemma 5.4 we have

$$
-\frac{\mathrm{d}}{\mathrm{d} r}\left(r^{d-1} \frac{\mathrm{d} \xi}{\mathrm{d} r}(r)\right)=\frac{m-1}{m}\left(r^{d-1} W(r)+d r^{d-1}\right) \quad \text { for } \quad r \in(0, \varrho),
$$

where $\varrho$ denotes the radius of the support of $W$ and $\xi:=W^{m-1}$. Introducing

$$
Q(r):=\int_{B(0, r)} W(x) \mathrm{d} x=\sigma_{d} \int_{0}^{r} W(z) z^{d-1} \mathrm{~d} z \quad \text { for } \quad r \in(0, \varrho),
$$

we integrate the previous differential equation to obtain

$$
-m r^{d-1} W(r)^{m-2} \frac{\mathrm{d} W}{\mathrm{~d} r}(r)=\frac{Q(r)}{\sigma_{d}}+r^{d} \quad \text { for } \quad r \in(0, \varrho) .
$$


Multiplying the above identity by $\sigma_{d} r W(r)$ and integrating over $(0, \infty)$ then lead us to the formula

$$
d\|W\|_{m}^{m}=\int_{0}^{\infty} r Q(r) W(r) \mathrm{d} r+M_{2}[W]
$$

As

$$
2 \sigma_{d} \int_{0}^{\infty} r Q(r) W(r) \mathrm{d} r=\mathcal{W}(W)
$$

by Newton's theorem [25, Theorem 9.7], we end up with the identity $(d-2) \mathcal{F}[W]=M_{2}[W]$ and (5.4) follows by the definition of $\nu_{M}$ and $\mathcal{G}$. We next multiply (5.3) by $2 W$ and integrate over $\mathbb{R}^{d}$ to obtain (5.5).

We next prove the following comparison result.

Lemma 5.6 Consider $M_{1} \in\left(0, M_{c}\right)$ and $M_{2} \in\left(0, M_{c}\right)$. For $i=1,2$ let $W_{i}$ be a minimiser of $\mathcal{G}$ in $\mathcal{Z}_{M_{i}}$ and denote by $\varrho_{i}$ the radius of its support (which is finite according to Lemma 5.4). If $W_{1}(0)>W_{2}(0)$ then $Q_{1}(r)>Q_{2}(r)$ for $r \in\left(0, \max \left\{\varrho_{1}, \varrho_{2}\right\}\right)$ where

$$
Q_{i}(r):=\int_{B(0, r)} W_{i}(x) \mathrm{d} x \quad \text { for } \quad r \in\left(0, \max \left\{\varrho_{1}, \varrho_{2}\right\}\right) \quad \text { and } \quad i=1,2 .
$$

Owing to Lemma 5.3 and Lemma 5.4, the proof of Lemma 5.6 is similar to that of $[26$, Lemma 10] to which we refer.

Proof of Theorem 5.2. Consider $M \in\left(0, M_{c}\right)$ and assume for contradiction that $\mathcal{G}$ has two minimisers $W_{1}$ and $W_{2}$ in $\mathcal{Z}_{M}$ with $W_{1}(0)>W_{2}(0)$. Denoting by $\varrho_{i}$ the radius of the support of $W_{i}$ and introducing

$$
Q_{i}(r):=\int_{B(0, r)} W_{i}(x) \mathrm{d} x
$$

for $r \in\left[0, \max \left\{\varrho_{1}, \varrho_{2}\right\}\right]$ and $i=1,2$, we infer from Lemma 5.6 that $Q_{1}(r)>Q_{2}(r)$ for all $r \in\left(0, \max \left\{\varrho_{1}, \varrho_{2}\right\}\right)$. Then $\varrho_{1} \leq \varrho_{2}$ and (5.4) warrants that

$$
2(m-1) \nu_{M}=\sigma_{d} \int_{0}^{\infty} r^{2} \frac{\mathrm{d}}{\mathrm{d} r}\left(Q_{i}-M\right)(r) \mathrm{d} r=\int_{0}^{\infty} 2 r\left(M-Q_{i}(r)\right) \mathrm{d} r
$$

for $i=1,2$. Consequently,

$$
\int_{0}^{\varrho_{2}} 2 r\left(Q_{1}-Q_{2}\right)(r) \mathrm{d} r=0
$$

which implies that $\varrho_{1}=\varrho_{2}$ and $Q_{1}=Q_{2}$, hence a contradiction.

Corollary 5.7 If $M \in\left(0, M_{c}\right)$ there exists a self-similar solution $U_{M}$ to (1.3) given by

$$
U_{M}(t, x)=\frac{1}{1+d t} W_{M}\left(\frac{x}{(1+d t)^{1 / d}}\right)
$$

where $W_{M}$ is the unique minimiser of $\mathcal{G}$ in $\mathcal{Z}_{M}$ given in Theorem 5.2.

Remark 5.8 Given $M \in\left(0, M_{c}\right)$, we expect that this self-similar solution attracts the dynamics of (1.3) for large times. Although we can prove that the $\omega$-limit set of the rescaled equation (5.1) consists of stationary solutions, we are yet lacking a uniqueness result to identify them as $W_{M}$. 
Acknowledgements.- The authors are grateful to Mohammed Lemou for pointing out [26] and to Pierre Raphaël for stimulating discussions. AB acknowledges the support of bourse Lavoisier and the KAUST investigator award. JAC acknowledges the support from DGI-MEC (Spain) project MTM2005-08024 and OT2008 programm at IPAM (UCLA). AB and JAC acknowledge partial support of the Acc. Integ./Picasso program HF2006-0198. We thank the Centre de Recerca Matemàtica (Barcelona) for providing an excellent atmosphere for research and financial support.

This paper is under the Creative Commons licence Attribution-NonCommercial-ShareAlike 2.5.

\section{References}

[1] L.A. Ambrosio, N. Gigli, And G. Savaré, Gradient flows in metric spaces and in the space of probability measures, Lectures in Mathematics, Birkhäuser, 2005.

[2] A.L. Bertozzi And M.C. Pugh, Long-wave instabilities and saturation in thin film equations, Comm. Pure Appl. Math., 51 (1998), pp. 625-661.

[3] A.L. Bertozzi And M.C. Pugh, Finite-time blow-up of solutions of some long-wave unstable thin film equations, Indiana Univ. Math. J., 49 (2000), pp. 1323-1366.

[4] P. Biler, G. Karch, P. Laurençot, and T. Nadzieja, The 8 8 -problem for radially symmetric solutions of a chemotaxis model in the plane, Math. Methods Appl. Sci., 29 (2006), pp. 1563-1583.

[5] A. Blanchet, V. Calvez, and J.A. Carrillo, Convergence of the mass-transport steepest descent scheme for the subcritical Patlak-Keller-Segel model, SIAM J. Numer. Anal., 46 (2008), pp. 691-721.

[6] A. Blanchet, J.A. Carrillo, and N. Masmoudi, Infinite time aggregation for the critical two-dimensional Patlak-Keller-Segel model, to appear in Comm. Pure Appl. Math.

[7] A. Blanchet, J. Dolbeault, and B. Perthame, Two-dimensional Keller-Segel model: optimal critical mass and qualitative properties of the solutions, Electron. J. Differential Equations 44 (2006), 32 pp. (electronic).

[8] V. Calvez and J.A. Carrillo, Volume effects in the Keller-Segel model: energy estimates preventing blow-up, J. Math. Pures Appl., 86 (2006), pp. 155-175.

[9] E. Carlen And M. Loss, Competing symmetries, the logarithmic HLS inequality and Onofri's inequality on $S^{n}$, Geom. Funct. Anal. 2 (1992), pp. 90-104.

[10] J.A. Carrillo, A. Jüngel, P.A. Markowich, G. Toscani, and A. Unterreiter, Entropy dissipation methods for degenerate parabolic problems and generalized Sobolev inequalities, Monatsh. Math., 133 (2001), pp. 1-82.

[11] J.A. Carrillo, R.J. McCann, and C. Villani, Kinetic equilibration rates for granular media and related equations: entropy dissipation and mass transportation estimates, Rev. Mat. Iberoamericana, 19 (2003), pp. 1-48.

[12] J.A. Carrillo, R.J. McCann, and C. Villani, Contractions in the 2-Wasserstein length space and thermalization of granular media, Arch. Ration. Mech. Anal., 179 (2006), pp. 217263. 
[13] J.A. Carrillo and G. Toscani, Asymptotic $L^{1}$-decay of solutions of the porous medium equation to self-similarity, Indiana Univ. Math. J., 49 (2000), pp. 113-142.

[14] T. Cazenave, Semilinear Schrödinger equations, Courant Lecture Notes in Mathematics, 10, New York University, Courant Institute of Mathematical Sciences, New York; American Mathematical Society, Providence, RI, 2003.

[15] P.-H. Chavanis And C. SiRe, Anomalous diffusion and collapse of self-gravitating Langevin particles in D dimensions, Phys. Rev. E, 69 (2004), 016116.

[16] L. Corrias, B. Perthame, And H. ZaAg, Global solutions of some chemotaxis and angiogenesis systems in high space dimensions, Milan J. Math., 72 (2004), pp. 1-28.

[17] J. Dolbeault, and B. Perthame, Optimal critical mass in the two-dimensional KellerSegel model in $\mathbb{R}^{2}$, C. R. Math. Acad. Sci. Paris, 339 (2004), pp. 611-616.

[18] B. Gidas, Wei-Ming Ni, and L. Nirenberg, Symmetry and related properties via the maximum principle, Comm. Math. Phys., 68 (1979), pp. 209-243.

[19] D. Gilbarg and N.S. Trudinger, Elliptic partial differential equations of second order, vol. 224 of Grundlehren der Mathematischen Wissenschaften [Fundamental Principles of Mathematical Sciences], Springer-Verlag, Berlin, second ed., 1983.

[20] D. Horstmann, From 1970 until present: the Keller-Segel model in chemotaxis and its consequences. I, Jahresber. Deutsch. Math.-Verein., 105 (2003), pp. 103-165.

[21] W. JäGer And S. Luckhaus, On explosions of solutions to a system of partial differential equations modelling chemotaxis, Trans. Amer. Math. Soc., 329 (1992), pp. 819-824.

[22] E.F. Keller And L.A. Segel, Initiation of slide mold aggregation viewed as an instability, J. Theor. Biol., 26 (1970), pp. 399-415.

[23] R. Kowalczyk, Preventing blow-up in a chemotaxis model, J. Math. Anal. Appl., 305 (2005), pp. 566-588.

[24] E.H. LiEB, Sharp constants in the Hardy-Littlewood-Sobolev and related inequalities, Ann. Math. (2), 118 (1983), pp. 349-374.

[25] E.H. Lieb And M. Loss, Analysis, vol. 14 of Graduate Studies in Mathematics, American Mathematical Society, Providence, RI, second ed., 2001.

[26] E.H. LiEB AND H.-T. YAU, The Chandrasekhar theory of stellar collapse as the limit of quantum mechanics, Comm. Math. Phys., 112 (1987), pp. 147-174.

[27] P.-L. Lions, The concentration-compactness principle in the calculus of variations. The locally compact case. I, Ann. Inst. H. Poincaré Anal. Non Linéaire, 1 (1984), pp. 109-145.

[28] R.J. McCann, A convexity principle for interacting gases, Adv. Math. 128, 1 (1997), pp. $153-179$.

[29] F. Merle And P. RAPhä̈L, On universality of blow-up profile for $L^{2}$ critical nonlinear Schrödinger equation, Invent. Math., 156 (2004), pp. 565-672.

[30] T. OGawa, Decay and asymptotic behavior of solutions of the Keller-Segel system of degenerate and nondegenerate type, Self-similar solutions of nonlinear PDE, 161-184, Banach Center Publ., 74, Polish Acad. Sci., Warsaw, 2006. 
[31] F. Отто, The geometry of dissipative evolution equations: the porous medium equation, Comm. Partial Differential Equations, 26 (2001), pp. 101-174.

[32] C. S. Patlak, Random walk with persistence and external bias, Bull. Math. Biophys., 15 (1953), pp. 311-338.

[33] D. Slepčev And M.C. Pugh, Selfsimilar blowup of unstable thin-film equations, Indiana Univ. Math. J., 54 (2005), pp. 1697-1738.

[34] Y. Sugryama, Global existence in sub-critical cases and finite time blow-up in super-critical cases to degenerate Keller-Segel systems, Differential Integral Equations 19 (2006), pp. 841876.

[35] Y. Sugiyama, Application of the best constant of the Sobolev inequality to degenerate KellerSegel models, Adv. Differential Equations 12 (2007), pp. 121-144.

[36] C. Sulem And P.L. Sulem, The nonlinear Schrödinger equation, Applied Mathematical Sciences 139, Springer-Verlag, New York, 1999.

[37] C.M. Topaz And A.L. Bertozzi, Swarming patterns in a two-dimensional kinematic model for biological groups, SIAM J. Appl. Math., 65 (2004), pp. 152-174.

[38] C.M. Topaz, A.L. Bertozzi, And M.A. Lewis, A nonlocal continuum model for biological aggregation, Bull. Math. Biol., 68 (2006), pp. 1601-1623.

[39] M. I. Weinstein, Nonlinear Schrödinger equations and sharp interpolation estimates, Comm. Math. Phys. 87 (1983), pp. 567-576.

[40] M. I. Weinstein, On the structure and formation of singularities in solutions to nonlinear dispersive evolution equations, Comm. Partial Differential Equations 11 (1986), pp. 545-565.

[41] V.E. Zakharov And A.B. Shabat, Exact theory of two-dimensional self-focusing and one-dimensional self-modulation of waves in nonlinear media, Ž. Èksper. Teoret. Fiz., 61 (1971), pp. 118-134. 